Article

\title{
The Effects of Dairy Intake on Insulin Resistance: A Systematic Review and Meta-Analysis of Randomized Clinical Trials
}

\author{
Kristen M. Sochol ${ }^{\dagger}$, Tanya S. Johns ${ }^{\dagger}$, Rupinder S. Buttar, Lovepreet Randhawa, Edeline Sanchez,

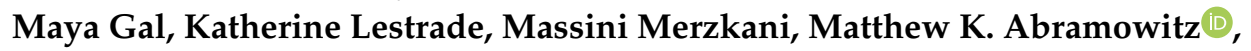 \\ Yasmin Mossavar-Rahmani and Michal L. Melamed * \\ Departments of Medicine and Epidemiology and Population Health, Albert Einstein College of Medicine, \\ Bronx, NY 10461, USA; kristen.sochol@gmail.com (K.M.S.); tjohns@montefiore.org (T.S.J.); \\ rubbybuttar@hotmail.com (R.S.B.); lvprt.randhawa90@gmail.com (L.R.); esanch01@syr.edu (E.S.); \\ mayagal1707@gmail.com (M.G.); katherine.lestrade@gmail.com (K.L.); massini00@hotmail.com (M.M.); \\ matthew.abramowitz@einstein.yu.edu (M.K.A.); yasmin.mossavar-rahmani@einstein.yu.edu (Y.M.-R.) \\ * Correspondence: michal.melamed@einstein.yu.edu; Tel.: +1-718-430-2304; Fax: +1-718-430-8963 \\ + These authors contributed equally to the study.
}

Received: 14 August 2019; Accepted: 12 September 2019; Published: 17 September 2019

\begin{abstract}
The incidence of type 2 diabetes mellitus (DM) has increased in the US over the last several years. The consumption of low-fat dairy foods has been linked with decreasing the risk of DM but studies have yet to show a clear correlation. We conducted a systematic review and meta-analysis of randomized clinical trials (RCTs) evaluating the effects of dairy intake on homeostatic model assessment of insulin resistance (HOMA-IR), waist circumference, and body weight. In MEDLINE and Embase, we identified and reviewed 49 relevant RCTs: 30 had appropriate data format for inclusion in the meta-analysis. Using the Review Manager 5 software, we calculated the pooled standardized mean differences comparing dairy dietary interventions to control for our outcomes of interest. For HOMA-IR (794 individuals), we found a mean difference of -1.21 (95\% CI -1.74 to $-0.67 ; p$-value $\left.<0.00001 ; I^{2}=92 \%\right)$. For waist circumference (1348 individuals), the mean difference was $-1.09 \mathrm{~cm}\left(95 \%\right.$ CI 1.68 to $-0.58 ; p$-value $\left.<0.00001 ; I^{2}=94 \%\right)$. For body weight ( 2362 individuals), the dairy intake intervention group weighed $0.42 \mathrm{~kg}$ less than control ( $p$-value $<0.00001 ; I^{2}=92 \%$ ). Our findings suggest that dairy intake, especially low-fat dairy products, has a beneficial effect on HOMA-IR, waist circumference, and body weight. This could impact dietary recommendations to reduce DM risk.
\end{abstract}

Keywords: dairy intake; insulin resistance; weight loss; waist circumference; diet; low-fat dairy

\section{Introduction}

There have been dramatic increases in the rates of obesity-associated diseases like type 2 diabetes mellitus (DM) potentially related to the modern lifestyle that is accompanied by excess food availability and reduced physical activity [1]. According to World Health Organization (WHO) 2016 report, the global prevalence of DM among adults over 18 years of age has risen from $4.7 \%$ in 1980 to $8.5 \%$ in 2014 [2]. In 2012, an estimated 1.5 million deaths were directly caused by diabetes mellitus [2]. Insulin resistance is a hallmark of type $2 \mathrm{DM}$ and likely precedes clinical DM by many years. An excess intake of calories and the resulting increased body fat can lead to the development of insulin resistance and type 2 DM [3].

Modifying dietary composition would be an appealing alternative to drug-based therapies to prevent the development of insulin resistance and DM. This would greatly help the more than 1.1 billion 
people worldwide who are overweight and at risk of DM [4,5]. One such potential food component is low-fat dairy products.

The data regarding the association of low-fat dairy products and body weight management is unclear. Animal and human in vitro studies consistently show an association of increased dairy intake with decreased lipogenesis and increased lipolysis, which could theoretically translate into body weight loss [6-8]. A recent meta-analysis by Geng et al. [9] suggested a beneficial effect of energy-restricted dairy consumption on body weight and composition. However, high dairy consumption in the absence of caloric restriction may be linked with increased body weight [9]. Observational studies have indicated a potential benefit of dairy products and their constituents on DM risk. Meta-analyses of cohort studies have demonstrated an inverse relationship between total dairy intake and type 2 DM risk [10,11]. A more recent large prospective cohort of $\sim 63,000$ Chinese men and women showed that daily intake of dairy products and daily milk consumption was associated with a modest decrease in DM risk [12]. In contrast, a Dutch prospective study on dairy product consumption showed no beneficial association with type 2 DM risk [13]. In the Framingham Offspring Study, a more complicated association between dairy intake and the risk of DM was found, with a protective association in participants with normal glycemia at baseline and only with certain dairy products [14]. In meta-analyses of observational studies on vitamin D and calcium intake (dairy constituents) in type 2 diabetes $[15,16]$ there was a significantly lower incidence of type 2 DM in the highest dairy versus lowest dairy intake groups. However, in a meta-analysis of randomized clinical trials (RCTs), vitamin D supplementation had no effect on glycemic outcomes except in patients with baseline glucose intolerance $[15,17]$. While vitamin $\mathrm{D}$ is one component of dairy products for some dairy products such as milk in the US, but not necessarily overseas, there may be effects of metabolomic components as well as nutrients such as calcium and proteins that may affect glycemic status. Therefore, we conducted a systematic review and meta-analysis of RCTs testing the effects of dairy supplementation on insulin resistance assessed through homeostasis model assessment-insulin resistance (HOMA-IR), waist circumference, and body weight which are established risk factors for type $2 \mathrm{DM}$.

\section{Materials and Methods}

\subsection{Search Strategy}

We conducted a search of MEDLINE and The Cochrane Library Central Register of Controlled Trials for RCTs from inception to July 2016 on the effect of dairy intake in adults related to insulin resistance or body mass index changes through weight loss. With the help of a medical librarian, we developed a search strategy using separate searches for the following medical subject headings: Dairy Product, in combination with each of the following Insulin Resistance, Weight Loss, Body Mass Index, Diet, and Reducing. In addition to Dairy Product, we also searched for individual components such as milk, butter, cheese, and yogurt. We limited the search to adults ( $\geq 18$ years), RCTs, and the English language. Additionally, we retrieved more articles from reference lists of recovered articles, review articles, and personal reference lists. All disagreements for study selection, data abstraction, and study quality assessments were resolved through discussion between the authors.

\subsection{Study Selection}

Four independent authors (K.M.S., M.L.M., E.S., R.S.B.) reviewed each study and used criteria for inclusion/exclusion of studies established prior to the literature search to assess each citation by title and abstract, or by reading the publication. Studies had to fulfill the following criteria for eligibility: (a) Randomization should be used to allocate patients in groups; (b) intervention of dairy supplementation with or without an energy deficit or caloric restriction; and (c) reported at least one of the following outcomes of interest: homeostasis model assessment-insulin resistance (HOMA-IR), waist circumference or body weight collected at baseline and follow-up. We also directly contacted 
authors to obtain additional data from published manuscripts in order to analyze as many studies as possible. All data used in this analysis was in aggregate, de-identified form.

\subsection{Data Abstraction}

Five authors (K.M.S., E.S., M.M., R.S.B., and T.S.J.) independently abstracted relevant data using predesigned data abstraction tables. Data abstracted included (a) participant demographics; (b) treatment information (type of intervention, dose, and duration); (c) number of participants; (d) duration of follow-up; (e) outcome data of interest at baseline and follow-up; and (f) body mass index.

\subsection{Study Quality and Risk of Bias Assessment}

Two authors (R.S.B. and L.R.) independently reviewed all included studies and rated them on the basis of 5 criteria: (1) Intention-to-treat analysis, (2) blinding of the study, (3) reporting of adherence, (4) sample size and power calculations reported, and (5) completeness of cohort follow-up. Risk of bias (ROB) assessment for each study was performed using a Cochrane risk of bias assessment tool modified to the five criteria. When three or more of the quality criteria were rated as having a high or unclear risk, the overall ROB was rated as high. If two or less of the quality items were rated as having a high or unclear risk, the overall ROB was rated as low.

\subsection{Publication Bias Assessment}

To assess for publication bias for each outcome, we constructed Begg and Mazumdar's funnel plots [18]. These funnel plots were drawn by using the standard error by standardized difference in means and confidence interval for all the studies included in our review.

\subsection{Statistical Analyses}

Standardized mean differences and their $95 \%$ confidence intervals (CI) were calculated using a random effects model as described by Dersimonian and Laird [19]. To assess heterogeneity across studies, we used the Cochran Q statistic based on the pooled standardized mean difference by Mantel-Haenszel, and measured inconsistency $\left(I^{2}\right.$; the percentage of total variance across studies attributable to heterogeneity rather than chance) of treatment effects across trials [20]. All $p$ values were two-tailed and a $p$-value $<0.05$ was considered statistically significant. All analyses were completed using Review Manager 5 (RevMan 5, Copenhagen, Denmark) computer software.

\subsection{Sensitivity Analyses}

We also performed sensitivity analyses to ascertain the effect on the point estimates and heterogeneity statistics for all three of the outcomes. This included limiting our analyses to studies assessed to have a low risk of bias and excluding studies that had a physical activity component as part of the intervention.

\section{Results}

\subsection{Study Selection}

From the initial search and personal reference lists, there were 2562 articles to be screened by title and abstract, or full text if necessary. Ninety-seven duplicates were removed, and 2415 studies were removed by abstract and title due to inappropriate study design, study population, or outcomes of interest (Figure 1). Forty-nine full text articles were reviewed from the initial search. Several of these articles reported their findings in formats inappropriate for the meta-analysis and the authors were contacted. The authors who responded and provided data with the correct format were included in the meta-analysis (Table 1). The remaining studies were discussed but not included in the meta-analysis (Supplement Table S1) [21-39]. 


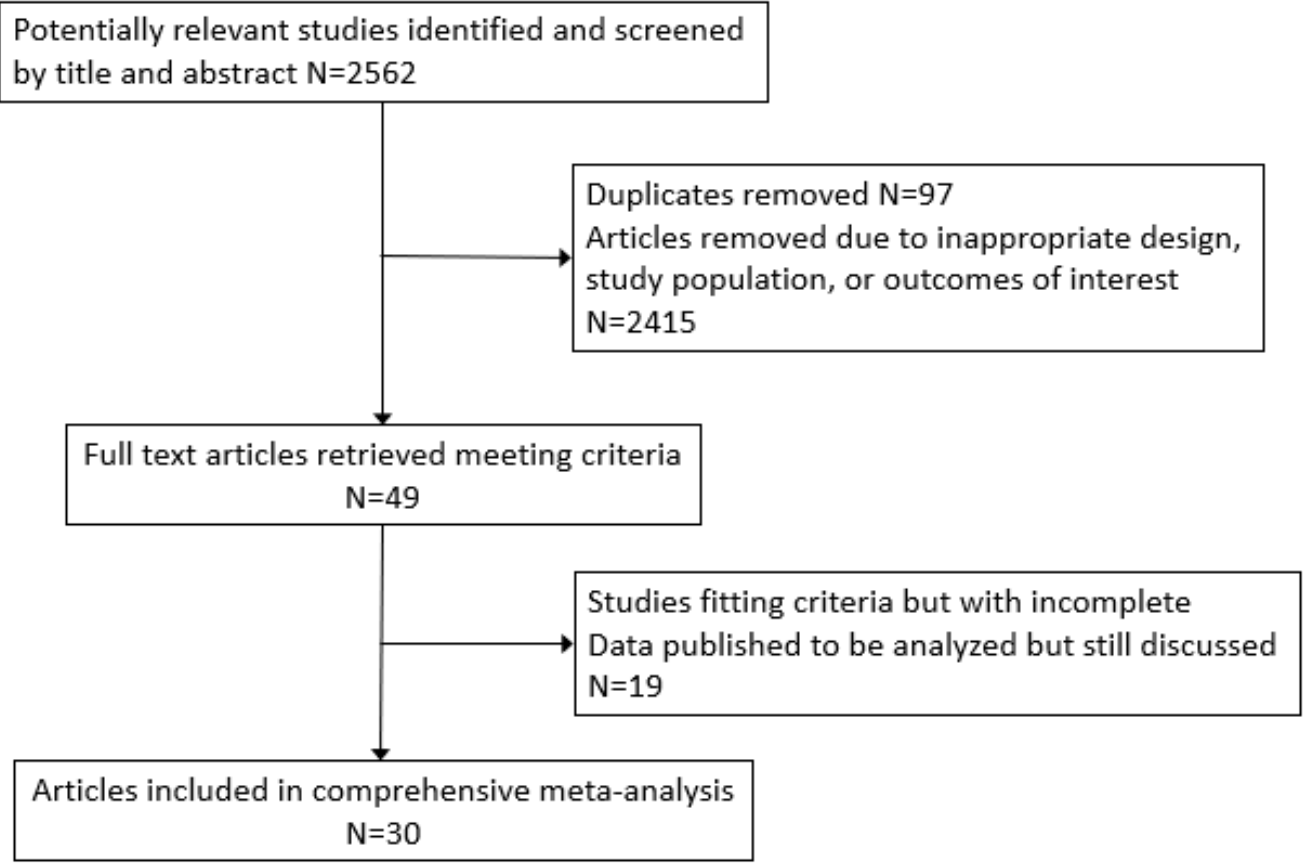

Figure 1. Flowchart of studies included in meta-analysis. 
Table 1. Randomized controlled trials of dairy intake included in the meta-analysis.

\begin{tabular}{|c|c|c|c|c|c|c|c|}
\hline $\begin{array}{c}\text { Study } \\
\text { Author, Year }\end{array}$ & Sample Size & Sex & $\begin{array}{c}\text { Age } \\
\text { Mean } \pm \text { SD or Range } \\
\text { (Years) }\end{array}$ & $\begin{array}{c}\text { BMI } \\
\text { Mean } \pm \text { SD or Range } \\
\left(\mathrm{kg} / \mathrm{m}^{2}\right)\end{array}$ & Country of Origin & $\begin{array}{l}\text { Study Duration } \\
\text { (Weeks) }\end{array}$ & $\begin{array}{c}\text { Findings } \\
\text { Intervention vs. Control }\end{array}$ \\
\hline Adamsson, 2011 [40] & $\begin{array}{l}\mathrm{I}=44 \\
\mathrm{C}=42\end{array}$ & $37 \% \mathrm{M} 63 \% \mathrm{~F}$ & $\begin{array}{l}I=52.6 \pm 7.8 \\
C=53.4 \pm 8.1\end{array}$ & $\begin{aligned} I & =26.3 \pm 3.3 \\
C & =26.5 \pm 3.3\end{aligned}$ & Sweden & 6 & $\begin{array}{c}\downarrow \text { Body weight } \\
\downarrow \text { BMI } \\
\downarrow \text { HOMA-IR }\end{array}$ \\
\hline Adamsson, 2015 [41] & $\begin{array}{l}\mathrm{I}=38 \\
\mathrm{C}=40\end{array}$ & $40 \% \mathrm{M} 60 \% \mathrm{~F}$ & $\begin{array}{l}\mathrm{I}=54.9 \pm 7.8 \\
\mathrm{C}=54.4 \pm 9\end{array}$ & $\begin{array}{l}\mathrm{I}=28.2 \pm 2.5 \\
\mathrm{C}=28.5 \pm 2.4\end{array}$ & Sweden & 12 & $\begin{array}{l}\leftrightarrow \text { HOMA -IR } \\
\leftrightarrow \text { Body weight }\end{array}$ \\
\hline Appel, 2003 [42] & $\begin{array}{l}I=269 \\
C=273\end{array}$ & $38 \% \mathrm{M} 62 \% \mathrm{~F}$ & $50 \pm 8.9$ & $18.5-45$ & United States & 26 & $\downarrow$ Body weight \\
\hline Asemi, 2013 [43] & $\begin{array}{l}I=16 \\
C=16\end{array}$ & $100 \% \mathrm{~F}$ & $\begin{array}{l}\mathrm{I}=27.7 \pm 5.4 \\
\mathrm{C}=29.7 \pm 5.6\end{array}$ & $\begin{array}{l}\mathrm{I}=30.2 \pm 4.6 \\
\mathrm{C}=29.7 \pm 3.3\end{array}$ & Iran & 4 & $\begin{array}{c}\downarrow \text { Insulin } \\
\downarrow \text { FPG } \\
\downarrow \text { HOMA-IR } \\
\leftrightarrow \text { BMI } \\
\leftrightarrow \text { Body weight }\end{array}$ \\
\hline Asemi, 2015 [44] & $\begin{array}{l}I=24 \\
C=24\end{array}$ & $100 \% \mathrm{~F}$ & $\begin{array}{l}I=29.4 \pm 6.2 \\
C=30.7 \pm 6.7\end{array}$ & $\begin{array}{l}\mathrm{I}=31.5 \pm 5.7 \\
\mathrm{C}=29.1 \pm 3.2\end{array}$ & Iran & 8 & $\begin{array}{c}\downarrow \text { HOMA-IR } \\
\downarrow \text { WC } \\
\downarrow \text { Body weight } \\
\downarrow \text { BMI }\end{array}$ \\
\hline Benatar, 2014 [45] & $\begin{array}{l}I=60 \\
C=60\end{array}$ & $36 \% \mathrm{M} 64 \% \mathrm{~F}$ & $\begin{array}{l}\mathrm{I}=46.3 \pm 10.5 \\
\mathrm{C}=48.6 \pm 12\end{array}$ & $\begin{array}{l}I=24.6 \pm 4.1 \\
C=24.3 \pm 4.0\end{array}$ & New Zealand & 4 & $\begin{array}{c}\leftrightarrow \text { WC } \\
\leftrightarrow \text { Body weight } \\
\leftrightarrow \text { Insulin } \\
\leftrightarrow \text { HOMA-IR }\end{array}$ \\
\hline Bendsen*, 2008 [46] & $\begin{array}{l}\mathrm{I}=11 \\
\mathrm{C}=11\end{array}$ & $45 \% \mathrm{M} 55 \% \mathrm{~F}$ & $18-50$ & $25-31$ & Denmark & 3 & $\leftrightarrow$ Body weight \\
\hline Bowen, 2005 [47] & $\begin{array}{l}\mathrm{I}=25 \\
\mathrm{C}=25\end{array}$ & $\begin{array}{l}60 \% \mathrm{M} \\
40 \% \mathrm{~F}\end{array}$ & $25-64$ & $25-35$ & Australia & 16 & $\begin{array}{c}\leftrightarrow \text { Body weight } \\
\leftrightarrow \text { HOMA-IR }\end{array}$ \\
\hline Demling, 2000 [48] & $\begin{array}{l}\mathrm{I} 1=14 \\
\mathrm{I} 2=14 \\
\mathrm{C}=10\end{array}$ & $100 \% \mathrm{M}$ & $28-40$ & $29 \pm 4.1$ & United States & 12 & $\begin{array}{c}\leftrightarrow \text { Body Weight } \\
\downarrow \text { \%Body fat } \\
\uparrow \% \text { Lean body mass }\end{array}$ \\
\hline Faghih, 2011 [49] & $\begin{array}{l}I=22 \\
C=20\end{array}$ & $100 \% \mathrm{~F}$ & $20-50$ & $25-40$ & Iran & 8 & $\begin{array}{c}\downarrow \text { Body weight } \\
\downarrow \text { BMI } \\
\downarrow \text { WC }\end{array}$ \\
\hline Farnsworth, 2003 [50] & $\begin{array}{l}\mathrm{I}=28 \\
\mathrm{C}=29\end{array}$ & $25 \% \mathrm{M} 75 \% \mathrm{~F}$ & $\begin{array}{l}\mathrm{I}=51.2 \pm 3.1 \\
\mathrm{C}=49.6 \pm 2.7\end{array}$ & $\begin{array}{l}\mathrm{I}=34.1 \pm 1.0 \\
\mathrm{C}=34.0 \pm 1.1\end{array}$ & Australia & 16 & $\begin{array}{c}\leftrightarrow \text { HOMA-IR } \\
\leftrightarrow \text { Abdominal fat } \\
\leftrightarrow \text { Body weight } \\
\leftrightarrow \text { Fat mass } \\
\uparrow \text { Lean mass }\end{array}$ \\
\hline
\end{tabular}


Table 1. Cont.

\begin{tabular}{|c|c|c|c|c|c|c|c|}
\hline $\begin{array}{c}\text { Study } \\
\text { Author, Year }\end{array}$ & Sample Size & Sex & $\begin{array}{c}\text { Age } \\
\text { Mean } \pm \text { SD or Range } \\
\text { (Years) }\end{array}$ & $\begin{array}{c}\text { BMI } \\
\text { Mean } \pm \text { SD or Range } \\
\left(\mathrm{kg} / \mathrm{m}^{2}\right) \\
\end{array}$ & Country of Origin & $\begin{array}{l}\text { Study Duration } \\
\text { (Weeks) }\end{array}$ & $\begin{array}{c}\text { Findings } \\
\text { Intervention vs. Contro }\end{array}$ \\
\hline Gunther, 2005 [51] & $\begin{array}{l}\mathrm{I} 1=48 \\
\mathrm{I} 2=45 \\
\mathrm{C}=42\end{array}$ & $100 \% \mathrm{~F}$ & $\begin{array}{l}\mathrm{I} 1=20.1 \pm 2.5 \\
\mathrm{I} 2=20.2 \pm 2.4 \\
\mathrm{C}=20.1 \pm 2.4\end{array}$ & $\begin{array}{l}\mathrm{I} 1=22.4 \pm 2.6 \\
\mathrm{I} 2=23.3 \pm 3.9 \\
\mathrm{C}=22.1 \pm 3.1\end{array}$ & United States & 52 & $\begin{array}{c}\leftrightarrow \text { Body weight } \\
\leftrightarrow \text { BMI } \\
\leftrightarrow \text { Body fat mass }\end{array}$ \\
\hline Harvey-Berino, 2005 [52] & $\begin{array}{l}\mathrm{I}=25 \\
\mathrm{C}=29\end{array}$ & $\begin{array}{l}8 \% \mathrm{M} \\
92 \% \mathrm{~F}\end{array}$ & $\begin{array}{l}\mathrm{I}=45.2 \pm 7.0 \\
\mathrm{C}=45.1 \pm 6.5\end{array}$ & $25-34.9$ & United States & 52 & $\begin{array}{c}\leftrightarrow \text { Body weight } \\
\leftrightarrow \text { \%Body fat } \\
\leftrightarrow \text { Body fat }\end{array}$ \\
\hline Josse, 2011 [53] & $\begin{array}{l}\mathrm{I} 1=30 \\
\mathrm{I} 2=30 \\
\mathrm{C}=30\end{array}$ & $100 \% \mathrm{~F}$ & $\begin{array}{l}\mathrm{I} 1=30 \pm 1.0 \\
\mathrm{I} 2=26 \pm 1.0 \\
\mathrm{C}=28 \pm 1.0\end{array}$ & $\begin{array}{l}\mathrm{I} 1=31.4 \pm 0.6 \\
\mathrm{I} 2=31.8 \pm 0.6 \\
\mathrm{C}=31.5 \pm 0.6\end{array}$ & Canada & 16 & $\begin{array}{l}\downarrow \text { Body fat } \\
\downarrow \text { Body weight }\end{array}$ \\
\hline Liu, 2010 [54] & $\begin{array}{l}\mathrm{I} 1=60 \\
\mathrm{I} 2=60 \\
\mathrm{C}=60\end{array}$ & $100 \% \mathrm{~F}$ & $\begin{array}{l}\mathrm{I} 1=55.9 \pm 3.8 \\
\mathrm{I} 2=56 \pm 4.4 \\
\mathrm{C}=56.4 \pm 4.7\end{array}$ & $\begin{array}{l}\mathrm{I} 1=24.6 \pm 3.4 \\
\mathrm{I} 2=24.8 \pm 3.8 \\
\mathrm{C}=24.1 \pm 3.8\end{array}$ & China & 26 & $\begin{array}{l}\stackrel{\leftrightarrow \text { HOMA-IR }}{\leftrightarrow} \text { Body weight }\end{array}$ \\
\hline Lukaszuk, 2007 [55] & $\begin{array}{l}\mathrm{I}=7 \\
\mathrm{C}=7\end{array}$ & $100 \% \mathrm{~F}$ & $18-45$ & $\begin{array}{l}\mathrm{I}=33.9 \pm 10.5 \\
\mathrm{C}=38.4 \pm 10.0\end{array}$ & United States & 8 & $\begin{array}{c}\leftrightarrow \text { Body weight } \\
\leftrightarrow \text { WC }\end{array}$ \\
\hline Manios, 2006 [56] & $\begin{array}{l}\mathrm{I}=39 \\
\mathrm{C}=36\end{array}$ & $100 \% \mathrm{~F}$ & $\begin{array}{l}\mathrm{I}=60.5 \pm 0.7 \\
\mathrm{C}=61.4 \pm 0.8\end{array}$ & $\begin{array}{l}\mathrm{I}=28.3 \pm 0.6 \\
\mathrm{C}=29.8 \pm 0.9\end{array}$ & Greece & 22 & $\downarrow \mathrm{BMI}$ \\
\hline Razavi Zade, 2016 [57] & $\begin{array}{l}I=30 \\
C=30\end{array}$ & $50 \% \mathrm{M} 50 \% \mathrm{~F}$ & $\begin{array}{c}C=42.8 \pm 10.6 \\
I=39.7 \pm 7.3\end{array}$ & $\begin{array}{l}C=28.3 \pm 3.3 \\
I=28.5 \pm 3.2\end{array}$ & Iran & 8 & $\begin{array}{c}\downarrow \text { Body weight } \\
\downarrow \text { BMI } \\
\downarrow \text { HOMA-IR }\end{array}$ \\
\hline Rosado, 2011 [58] & $\begin{array}{l}\mathrm{I}=43 \\
\mathrm{C}=41\end{array}$ & $100 \% \mathrm{~F}$ & $\begin{array}{l}I=34.9 \pm 5.6 \\
C=34.1 \pm 5.9\end{array}$ & $\begin{array}{l}\mathrm{I}=34.8 \pm 3.4 \\
\mathrm{C}=24.8 \pm 3.8\end{array}$ & Mexico & 16 & $\begin{array}{c}\leftrightarrow \text { WC } \\
\leftrightarrow \text { Body weight } \\
\leftrightarrow \text { BMI } \\
\leftrightarrow \text { \%Body fat } \\
\leftrightarrow \text { Body mass }\end{array}$ \\
\hline Smilowitz, 2011 [59] & $\begin{array}{l}I=22 \\
C=23\end{array}$ & $20 \% \mathrm{M} 80 \% \mathrm{~F}$ & $\begin{array}{l}I=25.1 \pm 5.3 \\
C=24.2 \pm 4.7\end{array}$ & $\begin{array}{l}\mathrm{I}=28.3 \pm 3.0 \\
\mathrm{C}=28.8 \pm 2.7\end{array}$ & United States & 12 & $\begin{array}{c}\leftrightarrow \text { Body weight } \\
\leftrightarrow \% \text { Body fat } \\
\leftrightarrow \% \text { Lean mass } \\
\leftrightarrow \text { WC }\end{array}$ \\
\hline Stancliffe, 2011 [60] & $\begin{array}{l}\mathrm{I}=20 \\
\mathrm{C}=20\end{array}$ & $\begin{array}{l}50 \% \mathrm{M} \\
50 \% \mathrm{~F}\end{array}$ & $\begin{aligned} I & =34.4 \pm 9.4 \\
C & =39.5 \pm 10.2\end{aligned}$ & $\begin{array}{l}\mathrm{I}=30.1 \pm 4.4 \\
\mathrm{C}=31.2 \pm 5.4\end{array}$ & United States & 12 & $\begin{array}{c}\leftrightarrow \text { Body weight } \\
\downarrow \% \text { Body fat } \\
\downarrow \text { WC } \\
\downarrow \text { HOMA-IR } \\
\downarrow \text { Insulin level }\end{array}$ \\
\hline
\end{tabular}


Table 1. Cont.

\begin{tabular}{|c|c|c|c|c|c|c|c|}
\hline $\begin{array}{c}\text { Study } \\
\text { Author, Year }\end{array}$ & Sample Size & Sex & $\begin{array}{c}\text { Age } \\
\text { Mean } \pm \text { SD or Range } \\
\text { (Years) }\end{array}$ & $\begin{array}{c}\text { BMI } \\
\text { Mean } \pm \text { SD or Range } \\
\left(\mathrm{kg} / \mathrm{m}^{2}\right)\end{array}$ & Country of Origin & $\begin{array}{l}\text { Study Duration } \\
\text { (Weeks) }\end{array}$ & $\begin{array}{c}\text { Findings } \\
\text { Intervention vs. Control }\end{array}$ \\
\hline Tanaka, 2014 [61] & $\begin{array}{l}I=102 \\
C=98\end{array}$ & $100 \% \mathrm{M}$ & $\begin{array}{l}\mathrm{I}=41.7 \pm 7.5 \\
\mathrm{C}=41.7 \pm 7.1\end{array}$ & $\begin{array}{l}\mathrm{I}=27.2 \pm 3.9 \\
\mathrm{C}=26.8 \pm 2.9\end{array}$ & Japan & 24 & $\begin{array}{c}\leftrightarrow \text { Body weight } \\
\leftrightarrow \text { \%Body fat } \\
\leftrightarrow \text { WC }\end{array}$ \\
\hline Thomas, 2011 [62] & $\begin{array}{l}\mathrm{I}=15 \\
\mathrm{C}=14\end{array}$ & $100 \% \mathrm{~F}$ & $29-45$ & $\begin{array}{l}I=29.4 \pm 2.0 \\
C=28.7 \pm 2.2\end{array}$ & United States & 16 & $\begin{array}{c}\leftrightarrow \text { Body weight } \\
\leftrightarrow \text { WC } \\
\leftrightarrow \% \text { Body fat }\end{array}$ \\
\hline Thompson, 2005 [63] & $\begin{array}{l}\mathrm{I} 1=30 \\
\mathrm{I} 2=31 \\
\mathrm{C}=29\end{array}$ & $14 \% \mathrm{M} 86 \% \mathrm{~F}$ & $25-70$ & $30-40$ & United States & 48 & $\begin{array}{c}\leftrightarrow \text { Body weight } \\
\leftrightarrow \text { Body fat } \\
\leftrightarrow \text { WC } \\
\downarrow \text { HOMA-IR }\end{array}$ \\
\hline Wennersberg, 2009 [64] & $\begin{array}{l}I=55 \\
C=54\end{array}$ & $33 \% \mathrm{M} 67 \% \mathrm{~F}$ & $\begin{aligned} \mathrm{M} & =51.2 \pm 8.1 \\
\mathrm{~F} & =56 \pm 7.4\end{aligned}$ & $\begin{array}{l}I=30.1 \pm 3.6 \\
C=30.0 \pm 3.3\end{array}$ & Norway & 26 & $\begin{array}{c}\leftrightarrow \text { Body weight } \\
\leftrightarrow \text { BMI } \\
\leftrightarrow \% \text { Body fat } \\
\leftrightarrow \text { WC } \\
\downarrow \text { HOMA-IR }\end{array}$ \\
\hline Zemel, 2004 [65] & $\begin{array}{l}\mathrm{I}=14 \\
\mathrm{C}=14\end{array}$ & $\begin{array}{l}17 \% \mathrm{M} \\
83 \% \mathrm{~F}\end{array}$ & $46 \pm 8$ & $30-39.9$ & United States & 24 & $\begin{array}{c}\downarrow \text { Body weight } \\
\downarrow \text { Body fat } \\
\downarrow \text { Insulin }\end{array}$ \\
\hline Zemel, 2005 [66] (Phase 1) & $\begin{array}{l}\mathrm{I}=17 \\
\mathrm{C}=17\end{array}$ & $\begin{array}{c}32 \% \mathrm{M} \\
68 \% \mathrm{~F}\end{array}$ & $\begin{array}{l}\mathrm{I}=42.5 \pm 2.6 \\
\mathrm{C}=41.3 \pm 2.7\end{array}$ & $\begin{array}{l}\mathrm{I}=34.1 \pm 0.7 \\
\mathrm{C}=34.9 \pm 0.8\end{array}$ & United States & 24 & $\begin{array}{c}\leftrightarrow \text { Body weight } \\
\downarrow \text { Body fat } \\
\uparrow \text { Lean mass } \\
\downarrow \text { WC } \\
\downarrow \text { Insulin }\end{array}$ \\
\hline Zemel, 2005 [66] (Phase 2) & $\begin{array}{l}\mathrm{I}=17 \\
\mathrm{C}=12\end{array}$ & $\begin{array}{l}14 \% \mathrm{M} \\
86 \% \mathrm{~F}\end{array}$ & $\begin{array}{l}\mathrm{I}=41.7 \pm 2.9 \\
\mathrm{C}=41.7 \pm 2.7\end{array}$ & $\begin{array}{l}I=35.6 \pm 0.7 \\
C=35.4 \pm 0.9\end{array}$ & United States & 24 & $\begin{array}{c}\downarrow \text { Body weight } \\
\downarrow \text { Body fat } \\
\uparrow \text { Lean mass } \\
\downarrow \text { WC } \\
\downarrow \text { Insulin } \\
\end{array}$ \\
\hline Zemel, 2005 [67] & $\begin{array}{l}\mathrm{I}=18 \\
\mathrm{C}=16\end{array}$ & $29 \% \mathrm{M} 71 \% \mathrm{~F}$ & $\begin{array}{l}\mathrm{I}=39 \pm 10 \\
\mathrm{C}=42 \pm 6\end{array}$ & $\begin{array}{l}\mathrm{I}=32.1 \pm 0.4 \\
\mathrm{C}=33.2 \pm 0.9\end{array}$ & United States & 12 & $\begin{array}{c}\downarrow \text { Body fat } \\
\downarrow W C\end{array}$ \\
\hline Zemel, 2008 [68] & $\begin{array}{l}I=169 \\
C=169\end{array}$ & ** & $\begin{array}{l}\mathrm{I}=39.9 \pm 7.2 \\
\mathrm{C}=40.7 \pm 6.8\end{array}$ & $\begin{array}{l}\mathrm{I}=34.4 \pm 3.2 \\
\mathrm{C}=34.6 \pm 3.1\end{array}$ & United States & 39 & $\begin{array}{c}\leftrightarrow \text { Body weight } \\
\leftrightarrow \text { BMI } \\
\leftrightarrow \text { WC }\end{array}$ \\
\hline Zemel, 2009 [69] & $\begin{array}{l}\mathrm{I}=32 \\
\mathrm{C}=38\end{array}$ & $23 \% \mathrm{M} 77 \% \mathrm{~F}$ & $\begin{array}{l}I=25.5 \pm 5.0 \\
C=25.3 \pm 4.9\end{array}$ & $\begin{array}{l}I=28.8 \pm 2.8 \\
C=29.3 \pm 2.8\end{array}$ & United States & 12 & $\begin{array}{c}\leftrightarrow \text { Body weight } \\
\leftrightarrow \text { Lean mass } \\
\downarrow \text { WC }\end{array}$ \\
\hline
\end{tabular}

* Cross-over design; ${ }^{* *}$ Data missing on sex; $\downarrow=$ decrease; $\uparrow=$ increase; $\leftrightarrow=$ no difference. Abbreviations: BMI, body mass index; C, control; F, female; HOMA-IR, homeostatic model assessment of insulin resistance; I, intervention; M, male; SD, standard deviation; WC, waist circumference. 
Thirty articles met inclusion criteria and were included in the meta-analysis [40-69] as seen in Table 1 . The total sample size was 2900 with $>50 \%$ female participants, but the distribution varied greatly across different studies. The mean age of subjects ranged from 18-63 years. Baseline study participant characteristics, intervention type, dose, and duration, along with specific study findings are shown in Table 1. The intervention descriptions and control group are outlined in Table 2 . The following control groups were identified: 1) no intervention (usual diet), 2) a diet similar in structure and energy intake with a habitual or low dose of dairy products ( $<2$ dairy products per day) or without the use of dairy, 3) placebo capsules, 4) an energy deficit diet, or 5) soy intake of a comparable energy intake. All except two studies tested the effects of high vs. low intakes of dairy $[45,47]$. Bowen et al. replaced the dairy products in controls with rice milk and soya while Benatar et al. eliminated dairy in controls with no substitution. A few of the studies also had a physical activity component as part of the overall intervention (Table 2) $[42,48,52,53,56,62,63,68]$.

Table 2. Description of intervention and control diet for studies in the meta-analysis.

\begin{tabular}{|c|c|c|}
\hline Study Author, Date & Dietary Intervention & Physical Activity Component \\
\hline $\begin{array}{l}\text { Adamsson, } \\
2011[40]\end{array}$ & $\begin{array}{c}\text { I = Nordic diet (low-fat dairy, fruit, berries, legumes, LDL-C lowering foods) } \\
\text { C = Habitual diet with usual physical activity }\end{array}$ & No \\
\hline $\begin{array}{l}\text { Adamsson, } \\
2015[41]\end{array}$ & $\begin{array}{l}\text { I = Breakfast consisting of oat bran, LFM, low-fat yogurt, jam, raisins, fruits } \\
\text { and berries, whole grain bread, low-fat spread, turkey meat, pickled herring, } \\
\text { or mackerel } \\
\qquad \mathrm{C}=\text { Habitual diet with recommendation for Nordic foods. }\end{array}$ & No \\
\hline Appel, 2003 [42] & $\begin{array}{c}\text { I = Diet rich in low-fat dairy, fruit, vegetables, whole grains } \\
((2-3 \text { servings/day of low-fat dairy), DASH diet: calcium } \sim 1250 \mathrm{mg} / \mathrm{day} \\
\mathrm{C}=\text { Habitual diet. Advised once about effect of lifestyle (exercise, and DASH } \\
\text { diet) on blood pressure }\end{array}$ & Yes \\
\hline $\begin{array}{c}\text { Asemi, } \\
2013[43]\end{array}$ & $\begin{array}{c}\text { I = Diet rich in low-fat dairy, fruit, vegetables, whole grains (DASH diet) in } \\
\text { proportions similar to control diet: calcium } \sim 1752 \mathrm{mg} / \text { day } \\
\mathrm{C}=\text { Recommended proportions of fat, protein, and carbs } \\
\sim 1082 \mathrm{mg} \text { calcium/day }\end{array}$ & No \\
\hline $\begin{array}{l}\text { Asemi, } \\
2015[44]\end{array}$ & $\begin{array}{c}\mathrm{I}=\text { Diet high in low-fat dairy ( } 3 \text { servings/day), fruits, lean meat, and } \\
\text { vegetables. (DASH diet: carb } 52 \% \text {, protein } 18 \% \text {, total fat } 30 \% \text { with BMI based } \\
\text { calories restriction in both groups, calcium } \sim 1714 \mathrm{mg} / \text { day } \\
\mathrm{C}=\text { Diet high in grains, simple sugars, dairy (2 servings daily), calcium } \\
\sim 1037 \mathrm{mg} / \text { day }\end{array}$ & No \\
\hline $\begin{array}{c}\text { Benatar, } \\
2014[45]\end{array}$ & $\begin{array}{l}\mathrm{I}=\text { High fat milk and dairy (extra } 2-3 \text { servings of dairy; } \sim 1300 \mathrm{mg} \text { calcium) } \\
\text { C }=\text { Dairy/day replaced with rice milk or soya }\end{array}$ & No \\
\hline $\begin{array}{l}\text { Bendsen, } \\
2008[46]\end{array}$ & $\begin{array}{l}\mathrm{I}=\text { High dairy diet from low-fat dairy products (calcium } 2300 \mathrm{mg} / \text { day) } \\
\qquad \mathrm{C}=\text { Low dairy diet (calcium } 700 \mathrm{mg} / \text { day) }\end{array}$ & No \\
\hline $\begin{array}{c}\text { Bowen, } \\
2005[47]\end{array}$ & $\begin{array}{l}\mathrm{I}=\text { High dairy intake from low-fat dairy products (calcium } 2400 \mathrm{mg} / \text { day) } \\
\qquad \mathrm{C}=\text { Low dairy intake (calcium } 500 \mathrm{mg} / \text { day) }\end{array}$ & No \\
\hline $\begin{array}{l}\text { Demling, } \\
2000[48]\end{array}$ & $\begin{array}{c}\mathrm{I} 1=75 \mathrm{gm} \text { casein hydrolysate plus control diet } \\
\mathrm{I} 2=75 \mathrm{gm} \text { whey hydrolysate plus control diet } \\
\mathrm{C}=\text { non lipogenic, hypocaloric diet alone ( } 80 \% \text { of predicted needs). }\end{array}$ & Yes \\
\hline $\begin{array}{l}\text { Faghih, } \\
2011[49]\end{array}$ & $\begin{array}{c}\text { I = High dairy intake with } 3 \text { services of low-fat milk daily ( } 3 \text { servings per day; } \\
\text { calcium) with } 1300 \mathrm{mg} / \text { day of calcium; } 500 \text { calorie energy deficit } \\
\mathrm{C}=\text { Low dairy intake with } 500-600 \mathrm{mg} / \text { day calcium; } 500 \text { calorie energy deficit }\end{array}$ & No \\
\hline $\begin{array}{l}\text { Farnsworth, } \\
2003[50]\end{array}$ & 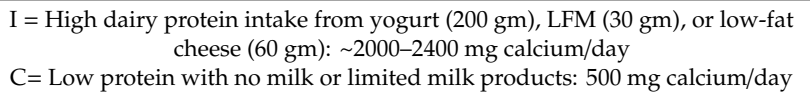 & No \\
\hline $\begin{array}{l}\text { Gunther, } \\
2005 \text { [51] }\end{array}$ & $\begin{array}{c}\mathrm{I} 1=\text { Substitute with dairy as source for } 1000-1100 \mathrm{mg} \text { calcium/day } \\
\mathrm{I} 2=\text { Substitute with dairy as source for } 1300-1400 \mathrm{mg} \text { calcium/day } \\
\mathrm{C}=\text { Maintain current dietary consumption of calcium }(\sim 700 \mathrm{mg} / \text { day }) \\
\text { (isocaloric diet for all groups) }\end{array}$ & No \\
\hline Harvey-Berino, 2005 [52] & $\begin{array}{c}\mathrm{I}=3-4 \text { servings dairy/day (equivalent to } 1200-1400 \mathrm{mg} \text { calcium) } \\
\mathrm{C}=1 \text { serving dairy/day (equivalent to } 500 \mathrm{mg} \text { calcium) } \\
\text { Dairy sources were milk cheese and yogurt }\end{array}$ & Yes \\
\hline $\begin{array}{c}\text { Josse, } \\
2011[53]\end{array}$ & $\begin{array}{l}\text { I1 }=3-4 \text { servings of dairy/day }(\sim 950 \mathrm{mg} \text { calcium/day }) \\
\mathrm{I} 2=6-7 \text { serving of dairy/day }(\sim 1650 \mathrm{mg} \text { calcium/day })) \\
\qquad \mathrm{C}=0-1 \text { serving dairy } / \text { day }\end{array}$ & Yes \\
\hline $\begin{array}{l}\text { Liu, } \\
2010[54]\end{array}$ & $\begin{array}{c}\mathrm{I} 1=15 \mathrm{~g} \text { milk protein on a daily basis in addition to usual diet } \\
\mathrm{I} 2=15 \mathrm{~g} \text { milk protein and } 100 \mathrm{mg} \text { isoflavones in addition to usual diet } \\
\mathrm{C}=15 \mathrm{~g} \text { soy protein and } 100 \mathrm{mg} \text { isoflavones in addition to usual diet }\end{array}$ & No \\
\hline $\begin{array}{l}\text { Lukaszuk, } \\
2007[55]\end{array}$ & $\begin{array}{c}\mathrm{I}=720 \mathrm{~mL} \text { skimmed milk/day }(\sim 900 \mathrm{mg} \text { calcium/day }) \\
\mathrm{C}=720 \mathrm{~mL} \text { soy milk/day }(\sim 1350 \mathrm{mg} \text { calcium/day })\end{array}$ & No \\
\hline
\end{tabular}


Table 2. Cont.

\begin{tabular}{|c|c|c|}
\hline Study Author, Date & Dietary Intervention & Physical Activity Component \\
\hline $\begin{array}{l}\text { Manios, } \\
2006[56]\end{array}$ & $\begin{array}{c}\mathrm{I}=\text { Low-fat dairy products (milk and yogurt) fortified with calcium and } \\
\text { vitamin } \mathrm{D}(\sim 1250 \mathrm{mg} \text { calcium/day }) \\
\mathrm{C}=\text { Regular diet }(\sim 730 \mathrm{mg} \text { calcium/day })\end{array}$ & Yes \\
\hline Razavi Zade, 2016 [57] & $\begin{array}{c}\mathrm{I}=\text { Energy restricted DASH diet consisted of } 52-55 \% \text { carbohydrates, } 16-18 \% \\
\text { proteins and } 30 \% \text { total fats ( } 1800 \mathrm{mg} \text { calcium/day). The DASH diet was rich } \\
\text { in fruits, vegetables, whole grains, and low-fat dairy products; and low in } \\
\text { saturated fats, cholesterol, refined grains, and sweets } \\
\text { C = Energy restricted regular diet with } 52-55 \% \text { carbohydrates, } 16-18 \% \\
\text { protein and } 30 \% \text { total fats }(\sim 1064 \mathrm{mg} \text { calcium/day). }\end{array}$ & No \\
\hline $\begin{array}{l}\text { Rosado, } \\
2011[58]\end{array}$ & $\begin{array}{l}\mathrm{I}=750 \mathrm{~mL} \text { of low-fat milk/ day in addition to an energy-restricted diet of } \\
\quad-500 \mathrm{kcal} / \mathrm{day}(\sim 1000 \mathrm{calcium} / \text { day }) \\
\\
\mathrm{C}=\text { Energy-restricted diet of }-500 \mathrm{kcal} / \text { day with no intake of milk }\end{array}$ & No \\
\hline $\begin{array}{l}\text { Smilowitz, } \\
2011[59]\end{array}$ & $\begin{array}{l}\mathrm{I}=\text { High dairy diet (placebo-supplemented) providing a } 2093 \mathrm{~kJ} / \text { day } \\
\text { deficit ( } 500 \mathrm{kcal} / \text { day) and containing } 3 \text { daily servings dairy products (milk, } \\
\text { cheese, and/or yogurt) to bring the total calcium intake to } 1400 \mathrm{mg} / \text { day } \\
\mathrm{C}=0-1 \text { serving of dairy products/day, } 500 \mathrm{mg} \text { calcium/day, and a daily } \\
\text { placebo supplement. Control diet providing a deficit ( } 500 \mathrm{kcal} / \text { day) }\end{array}$ & No \\
\hline $\begin{array}{l}\text { Stancliffe, } \\
2011[60]\end{array}$ & $\begin{array}{c}\mathrm{I}=\text { Adequate dairy diet }(3.5 \text { servings dairy daily }(\sim 1200 \mathrm{mg} \text { calcium/day }) \\
\mathrm{C}=\text { low-dairy diet }(<0.5 \text { serving/day } \sim \text { less than } 600 \mathrm{mg} \text { calcium/day })\end{array}$ & No \\
\hline $\begin{array}{l}\text { Tanaka, } \\
2014[61]\end{array}$ & $\begin{array}{l}\mathrm{I}=\text { Milk and dairy products }(400 \mathrm{~g} / \text { day }) \\
\qquad \mathrm{C}=\text { Regular diet }\end{array}$ & No \\
\hline $\begin{array}{l}\text { Thomas, } \\
2011[62]\end{array}$ & $\begin{array}{c}\text { I = 6-oz serving of fat-free yogurt (Yoplait Light Thick and Creamy } \sim 200 \mathrm{mg} \\
\text { calcium) containing } 418 \mathrm{~kJ} 20 \mathrm{~min} \text { before and immediately after each exercise } \\
\text { session } \\
\text { C = 6-oz serving of an isoenergetic placebo beverage containing } 25 \mathrm{~g} \text { of } \\
\text { carbohydrate, } 0 \mathrm{~g} \text { of fat, and } 0 \mathrm{~g} \text { of protein }\end{array}$ & Yes \\
\hline $\begin{array}{l}\text { Thompson, } \\
2005[63]\end{array}$ & $\begin{array}{l}\text { I1 }=4 \text { servings/day dairy }(\sim 1387 \mathrm{mg} \text { calcium/day) } \\
\text { I2 }=4 \text { servings/day dairy }(\sim 1439 \mathrm{mg} \text { calcium/day) with an increased amount } \\
\text { of fiber (through additional whole grains, fruits, and vegetables) and with a } \\
\text { reduction in glycemic index (foods with a glycemic index }>100 \text { were strongly } \\
\text { discouraged). } \\
\text { C }=2 \text { servings/day dairy ( } ~ \\
\text { and } 500 \% \text { mg calcium/day) with } 30 \% \text { fat, } 20 \% \text { protein, } \\
\text { calcium and fiber. Energy deficit of } 500 \text { calories }\end{array}$ & Yes \\
\hline $\begin{array}{l}\text { Wennersberg, } \\
2009[36]\end{array}$ & $\begin{array}{c}\mathrm{I}=3 \text { to } 5 \text { serving of dairy products in their diet daily including milk } \\
\text { containing } 0.5-3 \% \text { fat (one portion defined as } 200 \mathrm{~g} \text { milk), yogurt or sour milk } \\
(1.0-5.4 \% \text { fat, } 200-250 \mathrm{~g}) \text {, cream or creme fraiche }(12-40 \% \text { fat, }=75 \mathrm{~g}) \text {, cheese } \\
(15-30 \%, 15-40 \mathrm{~g}), \text { butter or butter containing spreads }(40-80 \% \mathrm{fat}, 3-10 \mathrm{~g}), \\
\text { cottage cheese }(2-8 \%, 0.5 \mathrm{dL}) \text {, and ice cream occasionally }(\sim 1150 \mathrm{mg} \\
\text { calcium/day) } \\
\text { C = habitual diet without changing the intake of dairy products }(\sim 625 \mathrm{mg} \\
\text { calcium/day) }\end{array}$ & No \\
\hline $\begin{array}{c}\text { Zemel, } \\
2004[65]\end{array}$ & $\begin{array}{c}\text { I = High dairy diet (1200 to } 1300 \mathrm{mg} \text { of dietary calcium/day supplemented } \\
\text { with placebo). } \\
\begin{array}{c}\text { C = Standard dairy diet (400 to } 500 \mathrm{mg} \text { of dietary calcium/day supplemented } \\
\text { with placebo). }\end{array}\end{array}$ & No \\
\hline $\begin{array}{l}\text { Zemel, } \\
2005[66] \\
\text { (Phase 1) }\end{array}$ & $\begin{array}{c}\mathrm{I}=\text { High dairy diet }(1200 \mathrm{mg} \text { calcium/day including } 3 \text { servings/day of dairy } \\
\text { with at least one in the form of fluid milk). } \\
\qquad \mathrm{C}=\text { Low dairy diet ( } 500 \mathrm{mg} / \text { day calcium with }<1 \text { serving/day). }\end{array}$ & No \\
\hline $\begin{array}{l}\text { Zemel, } \\
2005[66] \\
\text { (Phase 2) }\end{array}$ & $\begin{array}{c}\mathrm{I}=\text { High dairy diet }(1200 \mathrm{mg} \text { calcium/day including } 3 \text { servings/day of dairy } \\
\text { with at least one in the form of fluid milk) with } 500-\mathrm{kcal} / \mathrm{d} \text { deficit. } \\
\begin{array}{c}\mathrm{C}=0 \text { to } 1 \text { servings of low-fat dairy products per day, and containing a total of } \\
<500 \mathrm{mg} \text { calcium per day with } 500-\mathrm{kcal} / \text { day deficit. }\end{array}\end{array}$ & No \\
\hline $\begin{array}{c}\text { Zemel, } \\
2005[67]\end{array}$ & $\begin{array}{c}\mathrm{I}=\text { Yogurt diet providing a } 500 \mathrm{kcal} / \mathrm{day} \text { deficit and containing three daily } \\
\text { six-ounce servings of a commercial fat-free yogurt (Yoplait Light), to bring the } \\
\text { total calcium intake from } 500-1100 \mathrm{mg} / \mathrm{day} \text {. } \\
\mathrm{C}=0 \text { to } 1 \text { servings of dairy products/day and } 400-500 \mathrm{mg} \text { calcium per day. } \\
\text { The control diet incorporated as a placebo three daily servings of a sugar-free, } \\
\text { calcium-free, prepackaged flavored gelatin dessert containing } 10 \mathrm{kcal} / \mathrm{serving} \\
\text { with } 500 \mathrm{kcal} / \text { day deficit. }\end{array}$ & No \\
\hline $\begin{array}{c}\text { Zemel, } \\
2008[68]\end{array}$ & $\begin{array}{c}\text { I = Recommended dairy diet ( }>3 \text { servings/day of milk, cheese or yogurt). } \\
\qquad \text { C = Low dairy diet ( }<1 \text { dairy serving/day). }\end{array}$ & Yes \\
\hline $\begin{array}{c}\text { Zemel, } \\
2009[69]\end{array}$ & $\begin{array}{l}\mathrm{I}=\text { High dairy diet (placebo supplemented) containing three daily servings } \\
\text { dairy products (milk, cheese, and/or yogurt) substituted for other protein } \\
\text { sources in the diet, to bring the total calcium intake to } 1400 \mathrm{mg} / \text { day with a } 500 \\
\text { calories/day deficit. } \\
\text { C = } 0 \text { to } 1 \text { servings of dairy products/day, } 500 \mathrm{mg} \text { calcium/day, and a } \\
\text { daily placebo } \\
\text { (methyl-cellulose) supplement with a } 500 \text { calories/day deficit. }\end{array}$ & No \\
\hline
\end{tabular}

Abbreviations: C, control group; DASH, dietary approaches to stop hypertension; I, intervention group; LDL-C, low density lipoprotein cholesterol; LFM, low-fat milk. 


\subsection{Dairy Intake and HOMA-IR}

When combining all studies of dairy intake reporting HOMA-IR, there were fourteen interventions studied from twelve articles $[40,41,43-45,47,57,60,63-66]$. Of these, one study had two intervention arms tested against the same control [63]. For the meta-analysis, we used the same control data for both intervention arms. A study comparing a high dairy diet to a low dairy diet conducted two separate randomized trials: One trial included 34 patients with caloric maintenance (phase 1) and the other included 29 patients who were under caloric restriction (phase 2) [66]. Separate meta-analysis was performed for each phase. The pooled standardized difference in means between the dairy and placebo groups in HOMA-IR was $-1.21\left(95 \% \mathrm{CI}-1.74\right.$ to $-0.67 ; p$-value $<0.00001 ; I^{2}=92 \%$ ) (Figure 2). Increase in dairy intake was also found to be associated with improved oxidative stress markers and HOMA- IR $[43,60]$. Within the fourteen treatment interventions of dairy and HOMA-IR, eleven of the interventions included only subjects with BMI $>25 \mathrm{~kg} / \mathrm{m}^{2}[41,43,44,47,57,60,63-66]$. We combined these studies and the pooled standardized difference in means was -1.39 (95\% CI -2.03 to -0.75 ; $p$-value $<0.00001 ; I^{2}=92 \%$ ) (Figure 3 ). This is a slightly greater reduction in HOMA-IR compared to when including all participants from all studies.

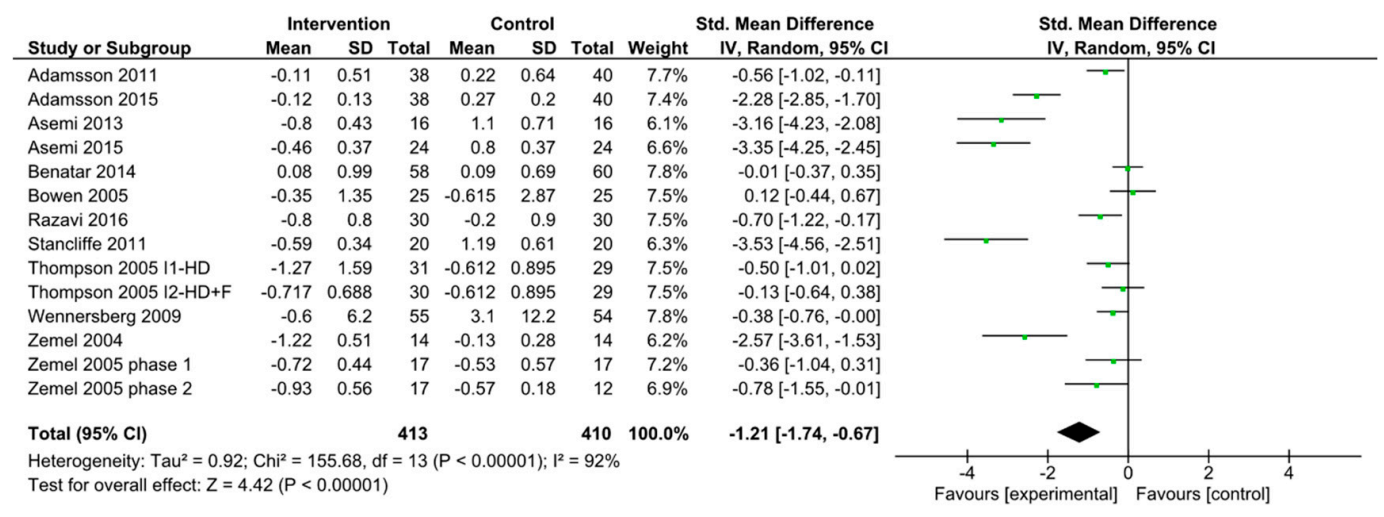

Figure 2. Forest plot of randomized clinical trials of dairy intake and HOMA-IR. Abbreviations: F, fiber; HD, high dairy diet.

\begin{tabular}{|c|c|c|c|c|c|c|c|c|c|c|}
\hline \multirow[b]{2}{*}{ Study or Subgroup } & \multicolumn{3}{|c|}{ Intervention } & \multicolumn{3}{|c|}{ Control } & \multicolumn{2}{|r|}{ Std. Mean Difference } & \multirow{2}{*}{\multicolumn{2}{|c|}{$\begin{array}{l}\text { Std. Mean Difference } \\
\text { IV, Random, } 95 \% \mathrm{CI}\end{array}$}} \\
\hline & Mean & SD & Total & Mean & SD & Total & Weight & IV, Random, $95 \% \mathrm{Cl}$ & & \\
\hline Adamsson 2015 & -0.12 & 0.13 & 38 & 0.27 & 0.2 & 40 & $8.7 \%$ & $-2.28[-2.85,-1.70]$ & & \\
\hline Asemi 2013 & -0.8 & 0.43 & 16 & 1.1 & 0.71 & 16 & $7.4 \%$ & $-3.16[-4.23,-2.08]$ & & \\
\hline Asemi 2015 & -0.46 & 0.37 & 24 & 0.8 & 0.37 & 24 & $7.9 \%$ & $-3.35[-4.25,-2.45]$ & & \\
\hline Bowen 2005 & -0.35 & 1.35 & 25 & -0.615 & 2.87 & 25 & $8.7 \%$ & $0.12[-0.44,0.67]$ & & \\
\hline Razavi 2016 & -0.8 & 0.8 & 30 & -0.2 & 0.9 & 30 & $8.8 \%$ & $-0.70[-1.22,-0.17]$ & & \\
\hline Stancliffe 2011 & -0.59 & 0.34 & 20 & 1.19 & 0.61 & 20 & $7.6 \%$ & $-3.53[-4.56,-2.51]$ & & \\
\hline Thompson 2005 |1-HD & -1.27 & 1.59 & 31 & -0.612 & 0.895 & 29 & $8.8 \%$ & $-0.50[-1.01,0.02]$ & & \\
\hline Thompson $2005 \mathrm{|} 2-\mathrm{HD}+\mathrm{F}$ & -0.717 & 0.688 & 30 & -0.612 & 0.895 & 29 & $8.8 \%$ & $-0.13[-0.64,0.38]$ & & \\
\hline Wennersberg 2009 & -0.6 & 6.2 & 55 & 3.1 & 12.2 & 54 & $9.1 \%$ & $-0.38[-0.76,-0.00]$ & & \\
\hline Zemel 2004 & -1.22 & 0.51 & 14 & -0.13 & 0.28 & 14 & $7.5 \%$ & $-2.57[-3.61,-1.53]$ & & \\
\hline Zemel 2005 phase 1 & -0.72 & 0.44 & 17 & -0.53 & 0.57 & 17 & $8.5 \%$ & $-0.36[-1.04,0.31]$ & & \\
\hline Zemel 2005 phase 2 & -0.93 & 0.56 & 17 & -0.57 & 0.18 & 12 & $8.2 \%$ & $-0.78[-1.55,-0.01]$ & & \\
\hline Total $(95 \% \mathrm{Cl})$ & & & 317 & & & 310 & $100.0 \%$ & $-1.39[-2.03,-0.75]$ & & \\
\hline $\begin{array}{l}\text { Heterogeneity: } \mathrm{Tau}^{2}=1.15 \\
\text { Test for overall effect: } Z=\end{array}$ & $\begin{array}{l}\mathrm{Chi}^{2}=13 \\
.25(P<C\end{array}$ & $\begin{array}{l}36.85, \mathrm{df} \\
0.0001)\end{array}$ & If $=11$ & $P<0.00$ & $001) ; 1^{2}$ & & & & $\begin{array}{ccc}-4 & -2 & 0 \\
\text { Favours [experimental] }\end{array}$ & $\begin{array}{lc}1 & 2 \\
& 2 \\
\text { Favours [control] }\end{array}$ \\
\hline
\end{tabular}

Figure 3. Forest plot of randomized clinical trials of dairy intake and HOMA-IR with BMI $>25$. Abbreviations: F, fiber; HD, high dairy diet.

\subsection{Dairy Intake and Waist Circumference}

Because changes in abdominal adiposity impacts insulin resistance, we examined whether dairy intake may affect changes in waist circumference [70,71]. We found 13 studies with 17 interventions comparing the effect of dairy on waist circumference $[44,45,49,53,55-57,60,61,63,64,66,68]$. Of these, 2 studies $[45,55]$ had controls with no dairy while the other studies did not completely exclude dairy from the control diet. Two studies had two intervention arms tested against the same control for which the meta-analysis was performed separately [53,63]. The pooled standardized difference in means 
was $-1.09 \mathrm{~cm}\left(95 \% \mathrm{CI}-1.61\right.$ to $-0.58 ; p$-value $\left.<0.00001 ; I^{2}=94 \%\right)$ comparing high diary intake to the control group (Figure 4).

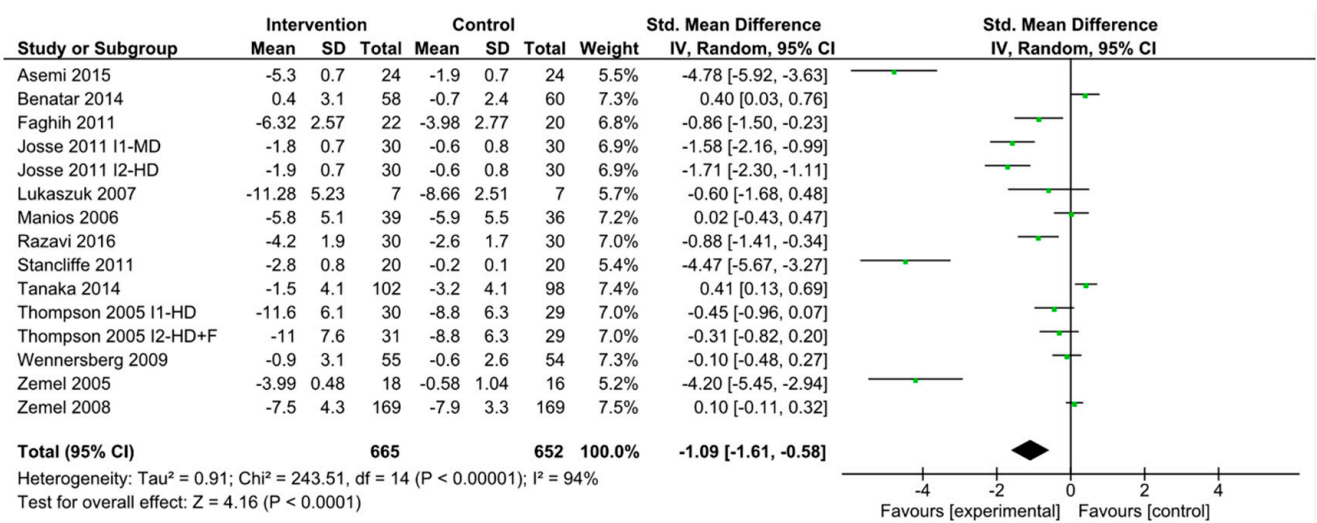

Figure 4. Forest plot of randomized clinical trials of dairy intake and waist circumference. Waist circumference change measured in $\mathrm{cm}$. Abbreviations: F, fiber; HD, high dairy diet; MD, moderate dairy diet.

\subsection{Dairy Intake and Body Weight}

We found 25 studies with 30 intervention arms [40-42,44-52,54,55,57,61-64,66,68,69,72,73]. Out of 25 studies, 4 studies had multiple intervention arms. For these studies, each intervention arm was treated as a separate trial $[48,51,54,63]$. Further, two more studies provided data for male and female participants separately, and were included in meta-analysis accordingly $[47,50]$. Another study was conducted in two phases but, accurate body weight change data was available for only the first phase. [66] Hence, the second phase was not included in the analysis. The pooled standardized difference in means was $-0.42 \mathrm{~kg}\left(95 \% \mathrm{CI}-0.72\right.$ to $-0.12 ; p$-value $\left.<0.00001 ; I^{2}=92 \%\right)$ comparing high dairy intake to the control group (Figure 5).

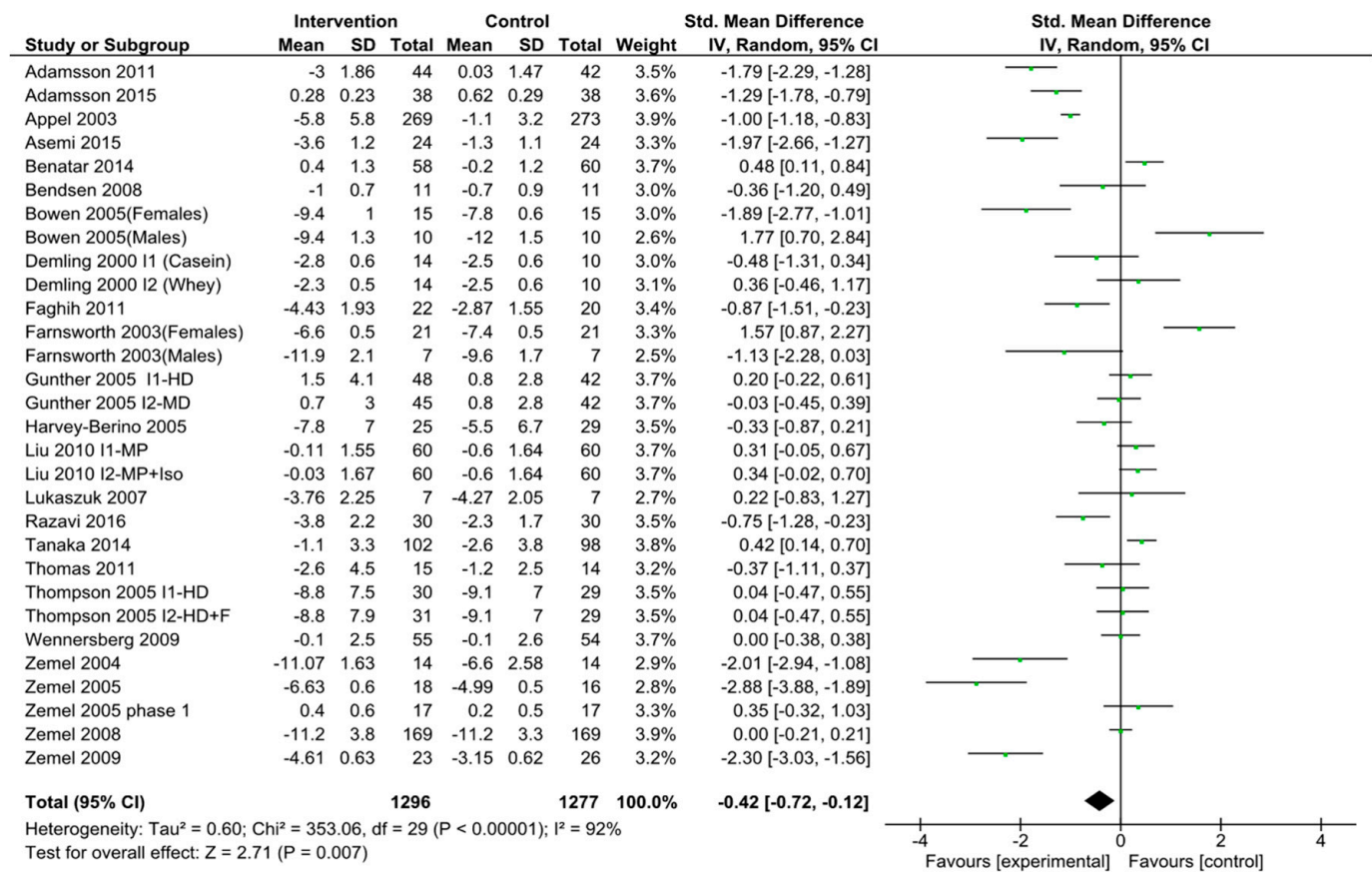

Figure 5. Forest plot of randomized clinical trials of dairy intake and body weight. Body weight change measured in Kg. Abbreviations: F, fiber; Iso, isoflavones; HD, high dairy diet; MD, moderate dairy diet; $\mathrm{MP}$, milk protein. 


\subsection{Risk of Bias}

The ROB assessment for each study is shown Figure 6 . A total of $26 \%$ of studies were assessed to have high risk of bias. For the vast majority studies, it was unclear if the investigators were blinded to study arms (Figure 7). The second most common cause of bias or unclear bias was whether the studies used intention-to-treat analysis. More than $90 \%$ of studies reported on completeness of follow-up/ attrition.

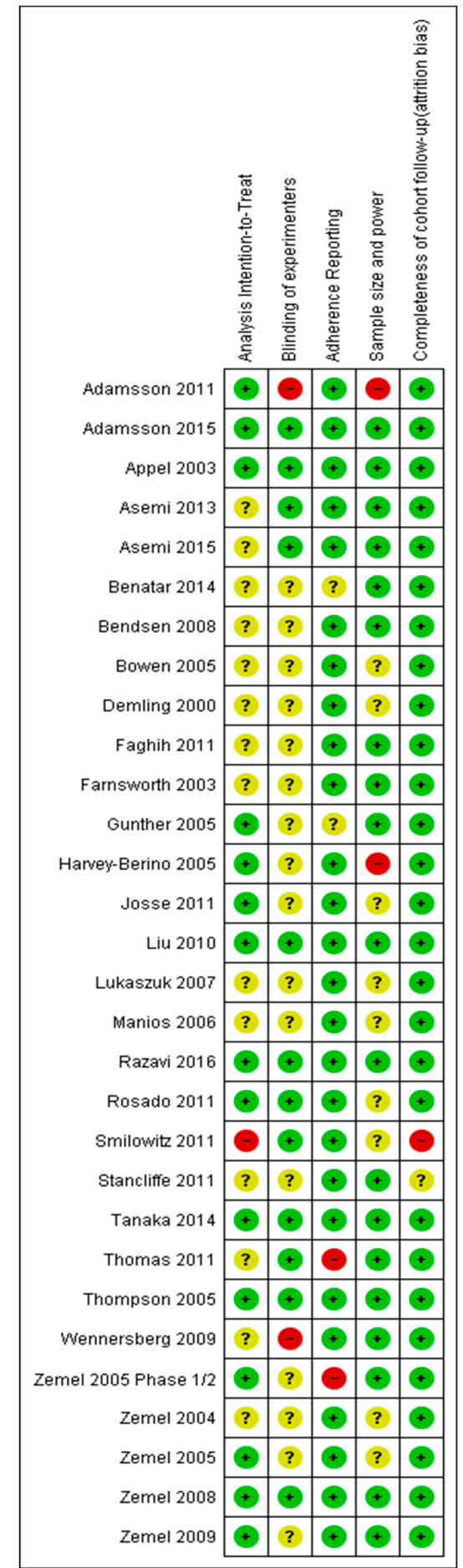

Figure 6. Risk of bias summary for all studies in the meta-analysis. Green= Low risk of bias, Yellow $=$ Unclear risk, Red $=$ High risk of bias. 


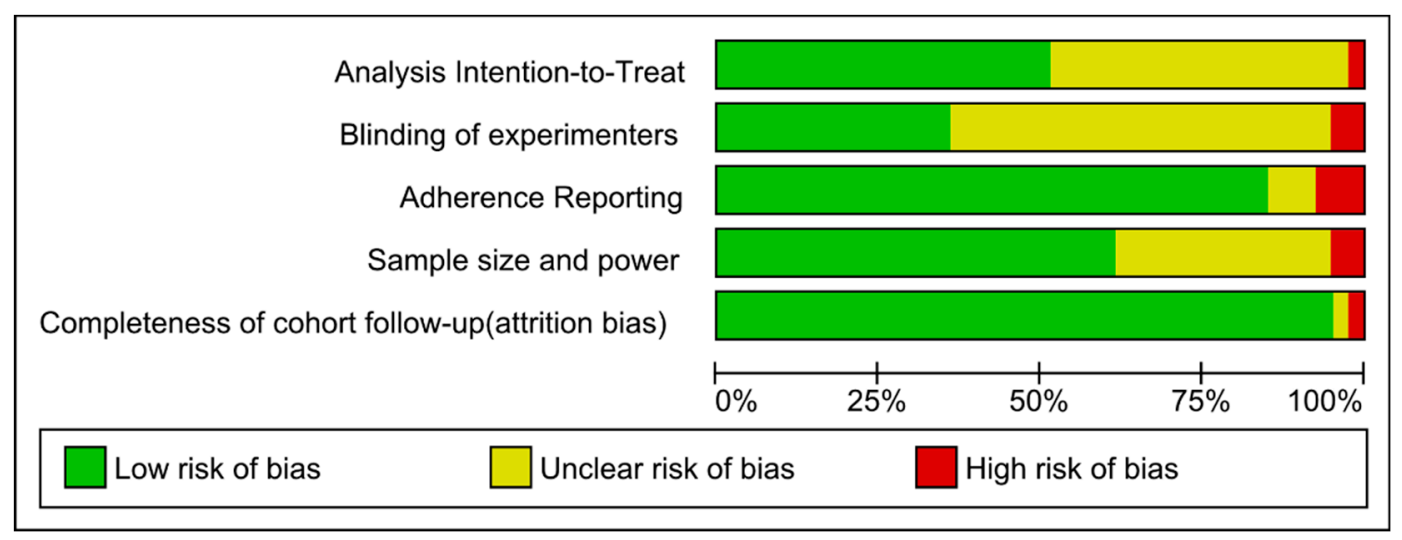

Figure 7. Risk of bias graph as percentages across all included studies.

\subsection{Publication Bias}

The funnel plots for all three outcomes did not suggest significant publication bias (Supplemental Figures S1-S3).

\subsection{Sensitivity Analyses}

Limiting our analysis to studies that were assessed to have low risk of bias did not significantly change the point estimates or heterogeneity statistics $\left(\mathrm{Q}\right.$ or $I^{2}$ ) for all three outcomes (Supplemental Figures S4-S6). Similarly excluding studies with a physical activity component did not significantly alter point estimates or heterogeneity statistics for all three outcomes (Supplemental Figures S7-S9).

\section{Discussion}

Our findings suggest that a diet which includes low-fat dairy products will decrease HOMA-IR in individuals, and therefore potentially decrease the risk of insulin resistance and type $2 \mathrm{DM}$. We also found a significantly lower waist circumference as well as body weight in individuals randomized to high dairy intake, suggesting that effects on HOMA-IR may be secondary to changes in body composition. We also found significant heterogeneity in the results which did not go away when restricting the analysis to only studies with low risk of bias or studies without a physical activity component as part of the intervention. The trials included have varying dietary dairy interventions, comparison groups (controls), and study populations which all likely contribute to the observed heterogeneity.

Our findings support previous observational studies that have shown an inverse association between diary intake and type 2 DM risk [10,11]. HOMA-IR is a reliable marker of insulin resistance [74]. Lower HOMA-IR levels have been linked to lower risks of several diseases including type 2 $\mathrm{DM}$, cardiovascular, cerebrovascular, and peripheral artery disease in large-scale prospective and cross-sectional studies such as the National Health and Nutrition Examination Survey (NHANES) [75]. In addition to HOMA-IR, waist circumference is another measure used to estimate the risk of type 2 DM [73]. Increased waist circumference appears to be an important contributor to the metabolic syndrome, which is a cluster of abnormalities including insulin resistance, dyslipidemia, and high blood pressure. Moreover, it more accurately reflects central obesity and visceral fat which are closely linked to the pathophysiology of insulin resistance [76].

There are several possible mechanisms to explain the proposed effects of low-fat dairy on insulin resistance. Calcium, vitamin D, casein, and whey proteins are all present in low-fat fortified dairy products and have been independently described as potential regulators of body fat, waist circumference, and insulin resistance. Zemel et al. have proposed a mechanism describing the relationship between low-calcium intake and body fat accumulation. Increases in body fat are associated with increased risk of insulin resistance [3]. Zemel et al. have also shown that decreases in extracellular calcium 
levels due to decreased calcium intake followed by a subsequent rise in calcitriol result in an increase in intracellular calcium levels. This, in turn, seems to promote energy storage in human adipocytes by stimulating the expression and activity of fatty acid synthase and by inhibiting lipolysis [77]. The calcium response sequence is located at the fatty acid synthase promoter region on the human genome. Increasing intracellular calcium has been shown to stimulate the expression and activity of fatty acid synthase. The increased expression of fatty acid synthase is coordinated with inhibition of lipolysis by the increased intracellular calcium levels [78,79]. Another possible mechanism through which calcium regulates body fat is that increased calcium intake has been shown to promote fat cell apoptosis. This apoptotic effect is through inhibition of UCP2 expression, a regulator of apoptosis [80]. Additionally, high intakes of calcium through supplements or dairy intake have been associated with diminished fat absorption in the gut because insoluble calcium fatty acid soaps or calcium bound to bile acids will increase fecal fat excretion [81,82].

Vitamin D is another constituent of dairy with several possible effects on insulin resistance through pancreatic beta-cell function, insulin sensitivity, and inflammation. Vitamin D has an effect on the insulin response to glucose stimulation [83]. This may be due to the fact that the circulating active form of vitamin $\mathrm{D}, 1$, 25-dihydroxyvitamin $\mathrm{D}$, binds directly to the beta-cell vitamin $\mathrm{D}$ receptor. In addition, activation of vitamin D may occur within the beta-cell by the 1-alpha-hydroxylase enzyme, which has been shown to be expressed in beta-cells [84], and this could explain the effect on insulin release. Vitamin D might directly influence insulin action by stimulating the expression of the insulin receptor on monocytes and thereby enhancing insulin responsiveness for glucose transport [85-88]. However, in a meta-analysis by Sarathy et al. on short-term vitamin D supplementation on glucose metabolism in end-stage kidney disease was found to be associated with lower fasting glucose levels with no change in fasting insulin levels [89]. In a recent intervention study of dairy intake vs. soy intake, dairy was shown to suppress the oxidative and inflammatory stress associated with overweight and obese subjects [39]. Vitamin D alone or as a component of dairy has been linked to the production and effects of cytokines and therefore may help to decrease insulin resistance [90-93].

Proteins in dairy foods such as casein and whey have been shown to have effects in reducing insulin resistance, hypertension, arterial stiffness, and LDL cholesterol in both human and animal studies [94-96]. In a double-blind, randomized, and controlled cross-over clinical trial of twelve subjects with type $2 \mathrm{DM}$ taken off their hypoglycemic therapy and studied after consuming medium calorie high protein mixed meals containing whey protein, casein, or a free amino acid mixture; the ingestion of casein and whey resulted in significantly higher beta-cell secretion and postprandial aminoacidemia than the ingestion of the free amino acid mixture [97]. In patients with type $2 \mathrm{DM}$, progressive deterioration in beta-cell function and mass are well established phenomena. Chronic stimulation of beta-cells, through postprandial aminoacidemia, may promote beta-cell proliferation and limit progressive deterioration. Postprandial aminoacidemia also has a stimulatory effect on the amplitude and possibly duration of muscle protein synthesis. The preservation of muscle can be beneficial in type $2 \mathrm{DM}$ to prevent declines in metabolic rate, which lead to fat mass accumulation and obesity $[98,99]$. A study by Demling et al. demonstrated that casein protein supplementation in weight training by individuals on a calorie-restricted diet leads to accelerated fat loss and lean mass gain [48]. These changes have been found to be associated with decreased insulin resistance.

Dairy intake may also promote weight loss and decrease insulin resistance by inducing satiation, thereby limiting intake of excess calories. In a meta-analysis of clinical trials by Onvani et al., the consumption of more than $500 \mathrm{~mL}$ of dairy products was significantly associated with increased satiety and decreased energy intake in the following meal [100].

There are limitations to the current analysis which should be mentioned. While BMI is often an important measure of risk for insulin resistance and type $2 \mathrm{DM}$, not enough of the studies reported adequate data on BMI to be included in the meta-analysis. However, results from NHANES III analysis comparing trends in BMI and waist circumference suggest that the harmful health consequences associated with obesity may be increasingly underestimated by trends in BMI alone and that waist 
circumference is an important independent risk factor to assess [101]. Another limitation of our study is that in evaluating food group (i.e., diary) we cannot discern the effects of individual foods (such as cheese, milk, yogurt) or components (such as calcium, vitamin D, or whey/ casein protein intake). This report was also limited because the many of the intervention studies examining the effects of dairy reported HOMA-IR insufficiently for meta-analysis. Furthermore, we did not have information on baseline glycemic status for the individuals included in the studies, which has been shown to influence the relationship between dairy intake and prediabetes or type 2 DM risk in the adults [14]. There was also significant heterogeneity among the dairy intervention trials included in our meta-analyses. Nonetheless, these findings provide valuable insight for healthcare providers as well as the general public on the possible positive impact of incorporating low-fat dairy into a healthy lifestyle.

\section{Conclusions}

Our findings suggest that dairy intake, especially low-fat dairy products, has a beneficial effect on HOMA-IR, waist circumference, and body weight. Some or all the mechanisms discussed above that are attributable to the components of low-fat dairy may work synergistically to improve insulin sensitivity, making it an appealing possible option for lowering the risk of insulin resistance and type 2 DM. Low-fat dairy products are easy to find and affordable in comparison to over the counter or prescription supplements. For a more definitive answer, larger scale randomized controlled trials of low-fat dairy intake and its impact on insulin resistance must be performed, especially in overweight and obese populations.

Supplementary Materials: The following are available online at http://www.mdpi.com/2072-6643/11/9/2237/s1, Table S1: Randomized Controlled Trials of Dairy Intake not included in the Meta-Analysis, Figure S1: Funnel plot of All Studies Evaluating Dairy intake and Body Weight, Figure S2: Funnel plot of All Studies Evaluating Dairy intake and Waist Circumference, Figure S3: Funnel plot of All Studies Evaluating Dairy intake and HOMA-IR, Figure S4: Forest Plot of Randomized Clinical Trials with Low Risk of Bias: Dairy Intake and HOMA-IR, Figure S5: Forest Plot of Randomized Clinical Trials with Low Risk of Bias: Dairy Intake and Waist Circumference, Figure S6: Forest Plot of Randomized Clinical Trials with Low Risk of Bias: Dairy Intake and Body Weight, Figure S7: Forest Plot of Randomized Clinical Trials without Physical Activity Component: Dairy Intake and HOMA-IR, Figure S8: Forest Plot of Randomized Clinical Trials without Physical Activity Component: Dairy Intake and Waist Circumference, Figure S9: Forest Plot of Randomized Clinical Trials without Physical Activity Component: Dairy Intake and Body Weight.

Author Contributions: K.M.S., M.M., M.L.M., R.S.B., and T.S.J. designed the research; E.S., K.M.S., K.L., L.R., M.G., M.M., M.L.M., and T.S.J. conducted the research; K.M.S., L.R., M.L.M., R.S.B., and T.S.J. analyzed the data; K.M.S., L.R., M.K.A., M.L.M., R.S.B., T.S.J., and Y.M.-R. wrote the paper; and K.M.S., L.R., M.L.M., and T.S.J. had primary responsibility for the final content. All authors read and approved the final manuscript.

Funding: This research was funded by National Institutes of Health (NIH) grants K23-078774 and K23-078774-S1 to Melamed, CTSA grants UL1RR025750, KL2RR025749 and TL1RR025748 from the National Center for Research Resources, a component of the NIH.

Acknowledgments: We would like thank Racheline Habousha and Aurelia Minuti for their help with the literature search and Michael Zemel, Joy Frestedt, Peter Clifton and Warren Thompson for providing us with additional data from their clinical trials.

Conflicts of Interest: The authors declare no conflict of interest.

\section{References}

1. Chatterjee, S.; Khunti, K.; Davies, M.J. Type 2 diabetes. Lancet 2017, 389, 2239-2251. [CrossRef]

2. Gojka, R. WHO Global report on diabetes: A summary. Int. J. Noncommun. Dis. 2016, 1, 3-8.

3. Frayn, K.N.; Tan, G.D.; Karpe, F. Adipose tissue: A key target for diabetes pathophysiology and treatment? Horm. Metab. Res. 2007, 39, 739-742. [CrossRef]

4. International Diabetes Federation. IDF Diabetes Atlas, 4th ed.; International Diabetes Federation: Brussels, Belgium, 2009.

5. Benjamin, S.M.; Valdez, R.; Geiss, L.S.; Rolka, D.B.; Narayan, K.M. Estimated number of adults with prediabetes in the US in 2000: Opportunities for prevention. Diabetes Care 2003, 26, 645-649. [CrossRef] [PubMed] 
6. Papakonstantinou, E.; Flatt, W.P.; Huth, P.J.; Harris, B.R. High dietary calcium reduces body fat content, digestibility of fat, and serum vitamin D in rats. Obes. Res. 2003, 11, 387-394. [CrossRef] [PubMed]

7. Shi, H.; Dirienzo, D.; Zemel, M.B. Effects of dietary calcium on adipocyte lipid metabolism and body weight regulation in energy-restricted aP2-agouti transgenic mice. FASEB J. 2001, 15, 291-293. [CrossRef] [PubMed]

8. Zemel, M.B.; Shi, H.; Greer, B.; Dirienzo, D.; Zemel, P.C. Regulation of adiposity by dietary calcium. FASEB J. 2000, 14, 1132-1138. [CrossRef] [PubMed]

9. Geng, T.; Qi, L.; Huang, T. Effects of Dairy Products Consumption on Body Weight and Body Composition Among Adults: An Updated Meta-Analysis of 37 Randomized Control Trials. Mol. Nutr. Food Res. 2018, 62. [CrossRef]

10. Gijsbers, L.; Ding, E.L.; Malik, V.S.; de Goede, J.; Geleijnse, J.M.; Soedamah-Muthu, S.S. Consumption of dairy foods and diabetes incidence: A dose-response meta-analysis of observational studies. Am. J. Clin Nutr. 2016, 103, 1111-1124. [CrossRef]

11. Schwingshackl, L.; Hoffmann, G.; Lampousi, A.M.; Knuppel, S.; Iqbal, K.; Schwedhelm, C.; Bechthold, A.; Schlesinger, S.; Boeing, H. Food groups and risk of type 2 diabetes mellitus: A systematic review and meta-analysis of prospective studies. Eur. J. Epidemiol. 2017, 32, 363-375. [CrossRef]

12. Talaei, M.; Pan, A.; Yuan, J.M.; Koh, W.P. Dairy intake and risk of type 2 diabetes. Clin. Nutr. 2018, 37, 712-718. [CrossRef] [PubMed]

13. Brouwer-Brolsma, E.M.; van Woudenbergh, G.J.; Elferink, S.J.O.; Singh-Povel, C.M.; Hofman, A.; Dehghan, A.; Franco, O.H.; Feskens, E.J. Intake of different types of dairy and its prospective association with risk of type 2 diabetes: The Rotterdam Study. Nutr. Metab. Cardiovasc. Dis. 2016, 26, 987-995. [CrossRef] [PubMed]

14. Hruby, A.; Ma, J.; Rogers, G.; Meigs, J.B.; Jacques, P.F. Associations of Dairy Intake with Incident Prediabetes or Diabetes in Middle-Aged Adults Vary by Both Dairy Type and Glycemic Status. J. Nutr. 2017, 147, 1764-1775. [CrossRef] [PubMed]

15. Mitri, J.; Muraru, M.D.; Pittas, A.G. Vitamin D and type 2 diabetes: A systematic review. Eur. J. Clin. Nutr. 2011, 65, 1005-1015. [CrossRef] [PubMed]

16. Pittas, A.G.; Lau, J.; Hu, F.B.; Dawson-Hughes, B. The role of vitamin D and calcium in type 2 diabetes. A systematic review and meta-analysis. J. Clin. Endocrinol. Metab. 2007, 92, 2017-2029. [CrossRef]

17. Seida, C.J.; Mitri, J.; Colmers, I.N.; Majumdar, S.R.; Davidson, M.B.; Edwards, A.L.; Hanley, D.A.; Pittas, A.G.; Tjosvold, L.; Johnson, J.A. Clinical review: Effect of vitamin D3 supplementation on improving glucose homeostasis and preventing diabetes: A systematic review and meta-analysis. J. Clin. Endocrinol. Metab. 2014, 99, 3551-3560. [CrossRef] [PubMed]

18. Begg, B.C.; Mazumdar, M. Operating characteristics of a rank correlation test for publication bias. Biometrics 1994, 50, 1088-1101. [CrossRef]

19. DerSimonian, R.; Laird, N. Meta-analysis in clinical trials. Control. Clin. Trials 1986, 7, 177-188. [CrossRef]

20. Cochran, W.G.; Cox, G.M. Experimental Design, 2nd ed.; John Wiley and Sons: New York, NY, USA, 1957; p. 615.

21. Anderson, J.W.; Hoie, L.H. Weight loss and lipid changes with low-energy diets: Comparator study of milk-based versus soy-based liquid meal replacement interventions. J. Am. College Nutri. 2005, 210-216. [CrossRef]

22. Lau, E.M.; Woo, J.; Lam, V.; Hong, A. Milk supplementation of the diet of postmenopausal Chinese women on a low calcium intake retards bone loss. J. Bone Miner. Res. 2001, 16, 1704-1709. [CrossRef]

23. Chee, W.S.; Suriah, A.R.; Chan, S.P.; Zaitun, Y.; Chan, Y.M. The effect of milk supplementation on bone mineral density in postmenopausal Chinese women in Malaysia. Osteoporos. Int. 2003, 14, 828-834. [CrossRef] [PubMed]

24. Sirtori, C.R.; Pazzucconi, F.; Colombo, L.; Battistin, P.; Bondioli, A.; Descheemaeker, K. Double-blind study of the addition of high-protein soya milk v. cows' milk to the diet of patients with severe hypercholesterolaemia and resistance to or intolerance of statins. Br. J. Nutr. 1999, 82, 91-96. [CrossRef] [PubMed]

25. Wagner, G.; Kindrick, S.; Hertzler, S.; DiSilvestro, R.A. Effects of various forms of calcium on body weight and bone turnover markers in women participating in a weight loss program. J. Am. Coll. Nutr. 2007, 26, 456-461. [CrossRef] [PubMed]

26. Azadbakht, L.; Mirmiran, P.; Esmaillzadeh, A.; Azizi, T.; Azizi, F. Beneficial effects of a Dietary Approaches to Stop Hypertension eating plan on features of the metabolic syndrome. Diabetes Care 2005, 28, 2823-2831. [CrossRef] [PubMed] 
27. Barr, S.I.; McCarron, D.A.; Heaney, R.P.; Dawson-Hughes, B.; Berga, S.L.; Stern, J.S.; Oparil, S. Effects of increased consumption of fluid milk on energy and nutrient intake, body weight, and cardiovascular risk factors in healthy older adults. J. Am. Diet. Assoc. 2000, 100, 810-817. [CrossRef]

28. Agrawal, R.P.; Jain, S.; Shah, S.; Chopra, A.; Agarwal, V. Effect of camel milk on glycemic control and insulin requirement in patients with type 1 diabetes: 2-years randomized controlled trial. Eur. J. Clin. Nutr. 2011, 65, 1048-1052. [CrossRef] [PubMed]

29. Buchowski, M.S.; Aslam, M.; Dossett, C.; Dorminy, C.; Choi, L.; Acra, S. Effect of dairy and non-dairy calcium on fecal fat excretion in lactose digester and maldigester obese adults. Int J. Obes. (Lond) 2010, 34, 127-135. [CrossRef] [PubMed]

30. Dugan, C.E.; Barona, J.; Fernandez, M.L. Increased dairy consumption differentially improves metabolic syndrome markers in male and female adults. Metab. Syndr. Relat. Disord. 2014, 12, 62-69. [CrossRef]

31. Liu, Z.; Qiu, L.; Chen, Y.M.; Su, Y.X. Effect of milk and calcium supplementation on bone density and bone turnover in pregnant Chinese women: A randomized controlled trail. Arch. Gynecol. Obstet. 2011, 283, 205-211. [CrossRef]

32. Maki, K.C.; Nieman, K.M.; Schild, A.L.; Kaden, V.N.; Lawless, A.L.; Kelley, K.M.; Rains, T.M. Sugar-sweetened product consumption alters glucose homeostasis compared with dairy product consumption in men and women at risk of type 2 diabetes mellitus. J. Nutr. 2015, 145, 459-466. [CrossRef]

33. Palacios, C.; Bertran, J.J.; Rios, R.E.; Soltero, S. No effects of low and high consumption of dairy products and calcium supplements on body composition and serum lipids in Puerto Rican obese adults. Nutrition 2011, 27, 520-525. [CrossRef] [PubMed]

34. Rideout, T.C.; Marinangeli, C.P.; Martin, H.; Browne, R.W.; Rempel, C.B. Consumption of low-fat dairy foods for 6 months improves insulin resistance without adversely affecting lipids or bodyweight in healthy adults: A randomized free-living cross-over study. Nutr. J. 2013, 12, 56. [CrossRef] [PubMed]

35. Van Meijl, L.E.; Mensink, R.P. Effects of low-fat dairy consumption on markers of low-grade systemic inflammation and endothelial function in overweight and obese subjects: An intervention study. Br. J. Nutr. 2010, 104, 1523-1527. [CrossRef] [PubMed]

36. Al-Naggar, R.A.; O, M.T.; Abdulghani, M. Low Fat Yogurt Reduce Weight: Randomized Clinical Trial from Malaysia. Res. J. Pharm. Biol. Chem. Sci. 2014, 5, 593.

37. Eagan, M.S.; Lyle, R.M.; Gunther, C.W.; Peacock, M.; Teegarden, D. Effect of 1-year dairy product intervention on fat mass in young women: 6-month follow-up. Obes. Obes. (Silver Spring) 2006, 14, 2242. [CrossRef]

38. Alonso, A.; Zozaya, C.; Vazquez, Z.; Martinez, J.A.; Martinez-Gonzalez, M.A. The effect of low-fat versus whole-fat dairy product intake on blood pressure and weight in young normotensive adults. J. Hum. Nutr. Diet 2009, 22, 336-342. [CrossRef]

39. Zemel, M.B.; X, S.; T, S.; B, W. Effects of dairy compared with soy on oxidative and inflammatory stress in overweight and obese subjects. Am. J. Clin. Nutr. 2010, 91, 16-22. [CrossRef] [PubMed]

40. Adamsson, V.; Reumark, A.; Fredriksson, I.B.; Hammarstrom, E.; Vessby, B.; Johansson, G.; Riserus, U. Effects of a healthy Nordic diet on cardiovascular risk factors in hypercholesterolaemic subjects: A randomized controlled trial (NORDIET). J. Intern. Med. 2011, 269, 150-159. [CrossRef]

41. Adamsson, V.; Reumark, A.; Marklund, M.; Larsson, A.; Riserus, U. Role of a prudent breakfast in improving cardiometabolic risk factors in subjects with hypercholesterolemia: A randomized controlled trial. Clin. Nutr. 2015, 34, 20-26. [CrossRef]

42. Appel, L.J.; Champagne, C.M.; Harsha, D.W.; Cooper, L.S.; Obarzanek, E.; Elmer, P.J.; Stevens, V.J.; Vollmer, W.M.; Lin, P.H.; Svetkey, L.P. Effects comprehensive lifestyle modification blood pressure control: Main results the PREMIER clinical trial. JAMA 2003, 289, 2083-2093.

43. Asemi, Z.; Samimi, M.; Tabassi, Z.; Sabihi, S.S.; Esmaillzadeh, A. A randomized controlled clinical trial investigating the effect of DASH diet on insulin resistance, inflammation, and oxidative stress in gestational diabetes. Nutrition 2013, 29, 619-624. [CrossRef] [PubMed]

44. Asemi, Z.; Esmaillzadeh, A. DASH diet, insulin resistance, and serum hs-CRP in polycystic ovary syndrome: A randomized controlled clinical trial. Horm. Metab. Res. 2015, 47, 232-238. [CrossRef] [PubMed]

45. Benatar, J.R.; Jones, E.; White, H.; Stewart, R.A. A randomized trial evaluating the effects of change in dairy food consumption on cardio-metabolic risk factors. Eur. J. Prev. Cardiol. 2014, 21, 1376-1386. [CrossRef] [PubMed] 
46. Bendsen, N.T.; Hother, A.L.; Jensen, S.K.; Lorenzen, J.K.; Astrup, A. Effect of dairy calcium on fecal fat excretion: A randomized crossover trial. Int. J. Obes. (Lond) 2008, 32, 1816-1824. [CrossRef] [PubMed]

47. Bowen, J.; Noakes, M.; Clifton, P.M. Effect of calcium and dairy foods in high protein, energy-restricted diets on weight loss and metabolic parameters in overweight adults. Int J. Obes. (Lond) 2005, 29, 957-965. [CrossRef] [PubMed]

48. Demling, R.H.; DeSanti, L. Effect of a hypocaloric diet, increased protein intake and resistance training on lean mass gains and fat mass loss in overweight police officers. Anna. Nutr. Metab. 2000, 44, 21-29. [CrossRef] [PubMed]

49. Faghih, S.; Abadi, A.R.; Hedayati, M.; Kimiagar, S.M. Comparison of the effects of cows' milk, fortified soy milk, and calcium supplement on weight and fat loss in premenopausal overweight and obese women. Nutr. Metab. Cardiovasc. Dis. 2011, 21, 499-503. [CrossRef]

50. Farnsworth, E.; Luscombe, N.D.; Luscombe, N.D.; Noakes, M.; Wittert, G.; Argyiou, E.; Clifton, P.M. Effect of a high-protein, energy-restricted diet on body composition, glycemic control, and lipid concentrations in overweight and obese hyperinsulinemic men and women. Am. J. Clin. Nutr. 2003, 78, 31-39. [CrossRef]

51. Gunther, C.W.; Legowski, P.A.; Lyle, R.M.; McCabe, G.P.; Eagan, M.S.; Peacock, M.; Teegarden, D. Dairy products do not lead to alterations in body weight or fat mass in young women in a 1-y intervention. Am. J. Clin. Nutr. 2005, 81, 751-756. [CrossRef]

52. Harvey-Berino, J.; Gold, B.C.; Lauber, R.; Starinski, A. The impact of calcium and dairy product consumption on weight loss. Obes. Res. 2005, 13, 1720-1726. [CrossRef]

53. Josse, A.R.; Atkinson, S.A.; Tarnopolsky, M.A.; Phillips, S.M. Increased consumption of dairy foods and protein during diet- and exercise-induced weight loss promotes fat mass loss and lean mass gain in overweight and obese premenopausal women. J. Nutr. 2011, 141, 1626-1634. [CrossRef] [PubMed]

54. Liu, Z.M.; Chen, Y.M.; Ho, S.C.; Ho, Y.P.; Woo, J. Effects of soy protein and isoflavones on glycemic control and insulin sensitivity: A 6-mo double-blind, randomized, placebo-controlled trial in postmenopausal Chinese women with prediabetes or untreated early diabetes. Am. J. Clin. Nutr. 2010, 91, 1394-1401. [CrossRef] [PubMed]

55. Lukaszuk, J.M.; Luebbers, P.; Gordon, B.A. Preliminary study: Soy milk as effective as skim milk in promoting weight loss. J. Am. Diet. Assoc. 2007, 107, 1811-1814. [CrossRef] [PubMed]

56. Manios, Y.; Moschonis, G.; Grammatikaki, E.; Katsaroli, I.; Kanelou, P.; Tanagra, S. Nutrition education in postmenopausal women: Changes in dietary and cardiovascular indices. Maturitas 2006, 55, 338-347. [CrossRef] [PubMed]

57. Razavi Zade, M.; Telkabadi, M.H.; Bahmani, F.; Salehi, B.; Farshbaf, S.; Asemi, Z. The effects of DASH diet on weight loss and metabolic status in adults with non-alcoholic fatty liver disease: A randomized clinical trial. Liver Int. 2016, 36, 563-571. [CrossRef]

58. Rosado, J.L.; Garcia, O.P.; Ronquillo, D.; Hervert-Hernandez, D.; Mdel, C.C.; Martinez, G.; Gutierrez, J.; Garcia, S. Intake of milk with added micronutrients increases the effectiveness of an energy-restricted diet to reduce body weight: A randomized controlled clinical trial in Mexican women. J. Am. Diet. Assoc. 2011, 111, 1507-1516. [CrossRef]

59. Smilowitz, J.T.; Wiest, M.M.; Teegarden, D.; Zemel, M.B.; German, J.B.; van Loan, M.D. Dietary fat and not calcium supplementation or dairy product consumption is associated with changes in anthropometrics during a randomized, placebo-controlled energy-restriction trial. Nutr. Metab. (Lond) 2011, 8, 67. [CrossRef]

60. Stancliffe, R.A.; Thorpe, T.; Zemel, M.B. Dairy attentuates oxidative and inflammatory stress in metabolic syndrome. Am. J. Clin. Nutr. 2011, 94, 422-430. [CrossRef]

61. Tanaka, S.; Uenishi, K.; Ishida, H.; Takami, Y.; Hosoi, T.; Kadowaki, T.; Orimo, H.; Ohashi, Y. A randomized intervention trial of 24-wk dairy consumption on waist circumference, blood pressure, and fasting blood sugar and lipids in Japanese men with metabolic syndrome. J. Nutr. Sci. Vitam. (Tokyo) 2014, 60, 305-312. [CrossRef]

62. Thomas, D.T.; Wideman, L.; Lovelady, C.A. Effects of a dairy supplement and resistance training on lean mass and insulin-like growth factor in women. Int. J. Sport Nutr. Exerc. Metab. 2011, 21, 181-188. [CrossRef]

63. Thompson, W.G.; Holdman, N.R.; Janzow, D.J.; Slezak, J.M.; Morris, K.L.; Zemel, M.B. Effect of energy-reduced diets high in dairy products and fiber on weight loss in obese adults. Obes. Res. 2005, 13, 1344-1353. [CrossRef] [PubMed] 
64. Wennersberg, M.H.; Smedman, A.; Turpeinen, A.M.; Retterstøl, K.; Tengblad, S.; Lipre, E.; Aro, A.; Mutanen, P.; Seljeflot, I.; Basu, S.; et al. Dairy products and metabolic effects in overweight men and women: Results from a 6-mo intervention study. Am. J. Clin. Nutr. 2009, 90, 960-968. [CrossRef] [PubMed]

65. Zemel, M.B.; Thompson, W.; Milstead, A.; Morris, K.; Campbell, P. Calcium and dairy acceleration of weight and fat loss during energy restriction in obese adults. Obes. Res. 2004, 12, 582-590. [CrossRef] [PubMed]

66. Zemel, M.B.; Richards, J.; Milstead, A.; Campbell, P. Effects of calcium and dairy on body composition and weight loss in African-American adults. Obes. Res. 2005, 13, 1218-1225. [CrossRef] [PubMed]

67. Zemel, M.B.; Richards, J.; Mathis, S.; Milstead, A.; Gebhardt, L.; Silva, E. Dairy augmentation of total and central fat loss in obese subjects. Int. J. Obes. 2005, 29, 391-397. [CrossRef]

68. Zemel, M.B.; Donnelly, J.E.; Smith, B.K.; Sullivan, D.K.; Richards, J.; Morgan-Hanusa, D.; Mayo, M.S.; Sun, X.; Cook-Wiens, G.; Bailey, B.W.; et al. Effects of dairy intake on weight maintenance. Nutr. Metab. (Lond) 2008, 5. [CrossRef]

69. Zemel, M.B.; Teegarden, D.; Loan, M.V.; Schoeller, D.A.; Matkovic, V.; Lyle, R.M.; Craig, B.A. Dairy-rich diets augment fat loss on an energy-restricted diet: A multicenter trial. Nutrients 2009, 1, 83-100. [CrossRef]

70. Wahrenberg, H.; Hertel, K.; Leijonhufvud, B.M.; Persson, L.G.; Toft, E.; Arner, P. Use of waist circumference to predict insulin resistance: Retrospective study. BMJ 2005, 330, 1363-1364. [CrossRef]

71. Racette, S.B.; Evans, E.M.; Weiss, E.P.; Hagberg, J.M.; Holloszy, J.O. Abdominal adiposity is a stronger predictor of insulin resistance than fitness among 50-95 year olds. Diabetes Care 2006, 29, 673-678. [CrossRef]

72. Asemi, Z.; Samimi, M.; Tabassi, Z.; Shakeri, H.; Sabihi, S.S.; Esmaillzadeh, A. Effects of DASH diet on lipid profiles and biomarkers of oxidative stress in overweight and obese women with polycystic ovary syndrome: A randomized clinical trial. Nutrition 2014, 30, 1287-1293. [CrossRef]

73. Jones, B.H.; Kim, J.H.; Zemel, M.B.; Woychik, R.P.; Michaud, E.J.; Wilkison, W.O.; Moustaid, N. Upregulation of adipocyte metabo- lism by agouti protein: Possible paracrine actions in yellow mouse obesity. Am. J. Physiol. 1996, 270, E192-E196. [PubMed]

74. Bonora, E.; Targher, G.; Alberiche, M.; Bonadonna, R.C.; Saggiani, F.; Zenere, M.B.; Monauni, T.; Muggeo, M. Homeostasis model assessment closely mirrors the glucose clamp technique in the assessment of insulin sensitivity: Studies in subjects with various degrees of glucose tolerance and insulin sensitivity. Diabetes Care 2000, 23, 57-63. [CrossRef] [PubMed]

75. Bonora, E.; Kiechl, S.; Willeit, J.; Oberhollenzer, F.; Egger, G.; Meigs, J.B.; Bonadonna, R.C.; Muggeo, M. Insulin resistance as estimated by homeostasis model assessment predicts incident symptomatic cardiovascular disease in caucasian subjects from the general population: The Bruneck study. Diabetes Care 2007, 30, 318-324. [CrossRef] [PubMed]

76. Després, J.P.; Lemieux, I. Abdominal obesity and metabolic syndrome. Nature 2006, 444, 881-887.

77. Claycombe, K.J.; Wang, Y.; Jones, B.H.; Kim, S.; Wilkison, W.O.; Zemel, M.B.; Chun, J.; Moustaid-Moussa, N. Transcriptional regulation of the adipocyte fatty acid synthase gene by agouti: Interaction with insulin. Physiol. Geniomics. 2000, 3, 157-162. [CrossRef] [PubMed]

78. Zemel, M. Nutritional and endocrine modulation of intracellular calcium. Implications in obesity, insulin resistance and hypertension. Mol. Cell Biochem. 1998, 188, 129-136. [CrossRef] [PubMed]

79. Xue, B.; Greenberg, A.G.; Kraemer, F.B.; Zemel, M.B. Mechanism of intracellular calcium ([Ca2+]i) inhibition of lipolysis in human adipocytes. FASEB J. 2001, 15, 2527-2529. [CrossRef]

80. Sun, X.Z.M. Role of uncoupling protein 2 (UCP2) expression and 1alpha, 25-dihydroxyvitamin D3 in modulating adipocyte apoptosis. FASEB J. 2004, 18, 1430-1432. [CrossRef]

81. Bland, R.; Markovic, D.; Hills, C.E.; Hughes, S.V.; Chan, S.L.; Squires, P.E.; Hewison, M. Expression of 25-hydroxyvitamin D3-1alpha-hydroxylase in pancreatic islets. J. Steroid Biochem. Mol. Biol. 2004, 89-90, 121-125. [CrossRef]

82. Jacobsen, R.; Lorenzen, J.K.; Toubro, S.; Krog-Mikkelsen, I.; Astrup, A. Effect of short-term high dietary calcium intake on 24-h energy expenditure, fat oxidation, and fecal fat excretion. Int J. Obes. (Lond) 2005, 29, 292-301. [CrossRef]

83. Billaudel, B.J.; Faure, A.G.; Sutter, B.C. Effect of 1,25 dihydroxyvitamin D3 on isolated islets from vitamin D3-deprived rats. Am. J. Physiol. 1990, 258, E643-648. [CrossRef] [PubMed]

84. Zehnder, D.; Bland, R.; Williams, M.C.; McNinch, R.W.; Howie, A.J.; Stewart, P.M.; Hewison, M. Extrarenal expression of 25-hydroxyvitamin d(3)-1 alpha-hydroxylase. J. Clin. Endocrinol. Metab. 2001, 86, 888-894. [PubMed] 
85. Welberg, J.W.; Monkelbaan, J.F.; de Vries, E.G.; Muskiet, F.A.; Cats, A.; Oremus, E.T.; Boersma-van Ek, W.; van Rijsbergen, H.; van der Meer, R.; Mulder, N.H.; et al. Effects of supplemental dietary calcium on quantitative and qualitative fecal fat excretion in man. Ann. Nutr. Metab. 1994, 38, 185-191. [CrossRef] [PubMed]

86. Maestro, B.; Campión, J.; Dávila, N.; Calle, C. Stimulation by 1,25-dihydroxyvitamin D3 of insulin receptor expression and insulin responsiveness for glucose transport in U-937 human promonocytic cells. Endocr. J. 2000, 47, 383-391. [CrossRef] [PubMed]

87. Zeitz, U.; Weber, K.; Soegiarto, D.W.; Wolf, E.; Balling, R.; Erben, R.G. Impaired insulin secretory capacity in mice lacking a functional vitamin D receptor. FASEB J. 2003, 17, 509-511. [CrossRef] [PubMed]

88. Bourlon, P.M.; Billaudel, B.; Faure-Dussert, A. Influence of vitamin D3 deficiency and 1,25 dihydroxyvitamin D3 on de novo insulin biosynthesis in the islets of the rat endocrine pancreas. J. Endocrinol. 1999, 160, 87-95. [CrossRef] [PubMed]

89. Sarathy, H.; Pramanik, V.; Kahn, J.; Abramowitz, M.K.; Meier, K.; Kishore, P.; Melamed, M.L. The effects of short-term vitamin D supplementation on glucose metabolism in dialysis patients: A systematic review and meta-analysis. Int. Urol. Nephrol. 2015, 47, 537-549. [CrossRef]

90. Timms, P.M.; Mannan, N.; Hitman, G.A.; Noonan, K.; Mills, P.G.; Syndercombe-Court, D.; Aganna, E.; Price, C.P.; Boucher, B.J. Circulating MMP9, vitamin D and variation in the TIMP-1 response with VDR genotype: Mechanisms for inflammatory damage in chronic disorders? QJM 2002, 95, 787-796. [CrossRef]

91. Pittas, A.G.; Mannan, N.; Hitman, G.A.; Noonan, K.; Mills, P.G.; Syndercombe-Court, D.; Aganna, E.; Price, C.P.; Boucher, B.J. The effects of calcium and vitamin D supplementation on blood glucose and markers of inflammation in nondiabetic adults. Diabetes Care 2007, 30, 980-986. [CrossRef]

92. Pradhan, A.D.; Manson, J.E.; Rifai, N.; Buring, J.E.; Ridker, P.M. C-reactive protein, interleukin 6, and risk of developing type 2 diabetes mellitus. JAMA 2001, 286, 327-334. [CrossRef]

93. Giulietti, A.; van Etten, E.; Overbergh, L.; Stoffels, K.; Bouillon, R.; Mathieu, C. Monocytes from type 2 diabetic patients have a pro-inflammatory profile. 1,25-Dihydroxyvitamin $\mathrm{D}(3)$ works as anti-inflammatory. Diabetes Res. Clin. Pract. 2007, 77, 47-57. [CrossRef] [PubMed]

94. Xiao, R.; Carter, J.A.; Linz, A.L.; Ferguson, M.; Badger, T.M.; Simmen, F.A. Dietary whey protein lowers serum C-peptide concentration and duodenal SREBP-1c mRNA abundance, and reduces occurrence of duodenal tumors and colon aberrant crypt foci in azoxymethane-treated male rats. J. Nutr. Biochem. 2006, 17, 626-634. [CrossRef] [PubMed]

95. Krissansen, G. Emerging health properties of whey proteins and their clinical implications. J. Am. Coll. Nutr. 2007, 26, 713S-723S. [CrossRef] [PubMed]

96. Prabhakar, V.R.; Venkatesan, N. Milk casein and its benefits on cardiovascular risk. Eur. Heart J. 2007, $28,139$. [CrossRef] [PubMed]

97. Tessari, P.; Kiwanuka, E.; Cristini, M.; Zaramella, M.; Enslen, M.; Zurlo, C.; Garcia-Rodenas, C. Slow versus fast proteins in the stimulation of beta-cell response and the activation of the entero-insular axis in type 2 diabetes. Diabetes Metab. Res. Rev. 2007, 23, 378-385. [CrossRef] [PubMed]

98. Wilkinson, S.B.; Tarnopolsky, M.A.; Macdonald, M.J.; Macdonald, J.R.; Armstrong, D.; Phillips, S.M. Consumption of fluid skim milk promotes greater muscle protein accretion after resistance exercise than does consumption of an isonitrogenous and isoenergetic soy-protein beverage. Am. J. Clin. Nutr. 2007, 85, 1031-1040. [CrossRef] [PubMed]

99. Phillips, S.M.; Tang, J.E.; Moore, D.R. The role of milk- and soy-based protein in support of muscle protein synthesis and muscle protein accretion in young and elderly persons. J. Am. Coll. Nutr. 2009, 28, 343-354. [CrossRef] [PubMed]

100. Onvani, S.; Haghighatdoost, F.; Surkan, P.J.; Azadbakht, L. Dairy products, satiety and food intake: A meta-analysis of clinical trials. Clin. Nutr. 2017, 36, 389-398. [CrossRef] [PubMed]

101. Walls, H.L.; Stevenson, C.E.; Mannan, H.R.; Abdullah, A.; Reid, C.M.; McNeil, J.J.; Peeters, A. Comparing Trends in BMI and Waist Circumference. Obesity Silver Spring. Obes. Res. 2010, 19, 216-219. [CrossRef] [PubMed]

(C) 2019 by the authors. Licensee MDPI, Basel, Switzerland. This article is an open access article distributed under the terms and conditions of the Creative Commons Attribution (CC BY) license (http://creativecommons.org/licenses/by/4.0/). 